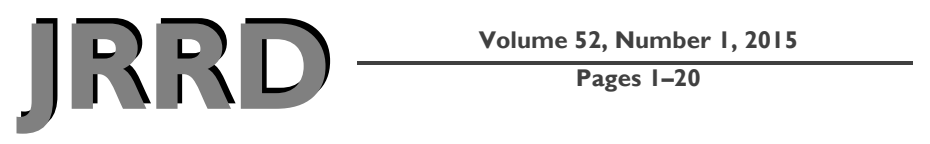

\title{
Spinal, pelvic, and hip movement asymmetries in people with lower-limb amputation: Systematic review
}

\author{
Hemakumar Devan, MPhty; ${ }^{*}$ Allan Carman, PhD; ${ }^{2}$ Paul Hendrick, PhD; ${ }^{3}$ Leigh Hale, PhD; ${ }^{1}$ Daniel Cury \\ Ribeiro, $\mathbf{P h D}^{\mathbf{1}}$ \\ ${ }^{1}$ Centre for Health, Activity, and Rehabilitation Research, School of Physiotherapy, University of Otago, Dunedin, New \\ Zealand; ${ }^{2}$ Sports Performance Research Institute New Zealand, Auckland University of Technology, Auckland, New \\ Zealand; ${ }^{3}$ Division of Physiotherapy and Rehabilitation Sciences, University of Nottingham, Nottingham, United Kingdom
}

\begin{abstract}
Following amputation, people with transfemoral amputation (TFA) and transtibial amputation (TTA) adapt with asymmetrical movements in the spinal and lower-limb joints. The aim of this review is to describe the trunk, lumbopelvic, and hip joint movement asymmetries of the amputated limb of people with TFA and TTA during functional tasks as compared with the intact leg and/or referent leg of nondisabled controls. Electronic databases were searched from inception to February 2014. Studies with kinematic data comparing (1) amputated and intact leg and (2) amputated and referent leg of nondisabled controls were included (26 articles). Considerable heterogeneity in the studies precluded data pooling. During stance phase of walking in participants with TFA, there is moderate evidence for increased trunk lateral flexion toward the amputated limb as compared with the intact leg and increased anterior pelvic tilt as compared with nondisabled controls. None of the studies investigated spinal kinematics during other functional tasks such as running, ramp walking, stair climbing, or obstacle crossing in participants with TFA or TTA. Overall, persons with TFA adapt with trunk and pelvic movement asymmetries at the amputated limb to facilitate weight transfer during walking. Among participants with TTA, there is limited evidence of spinal and pelvic asymmetries during walking.
\end{abstract}

Key words: activity of daily living, adaptation, amputation, biomechanics, compensation, functional task, kinematics, lower limb, transfemoral, transtibial.

\section{INTRODUCTION}

Lower-limb amputation, including transfemoral amputation (TFA) and transtibial amputation (TTA), is increasingly common secondary to vascular and nonvascular etiology [1-2]. Owing to the lack of intact knee and/ or ankle joints, persons with TFA and TTA compensate by increased loading on the intact leg as compared with the amputated leg during walking [3-5]. Further, the mechanical limitations of the prosthesis to fully weight bear on the amputated limb and the loss of lower-limb musculature leads to compensatory movements in the hip [6], pelvis [7], and trunk [8-9] segments during walking. Such compensatory movements are asymmetric in nature, with either increased or decreased motion occurring in the joints of the amputated limb as compared with the intact limb and/or referent limb of nondisabled controls [3,5,9]. The terms "movement adaptations" [3,6], "compensatory movements" [10-11], and "asymmetrical movements" [5,9]

\footnotetext{
Abbreviations: LBP $=$ low back pain, TFA $=$ transfemoral amputation, TTA $=$ transtibial amputation.

*Address all correspondence to Hemakumar Devan, MPhty; Centre for Health, Activity, and Rehabilitation Research, School of Physiotherapy, University of Otago, Dunedin, New Zealand; +64-3-479-9619.

Email: hemakumar.devan@gmail.com

http://dx.doi.org/10.1682/JRRD.2014.05.0135
} 
are often used interchangeably in the amputation literature. While movement asymmetries are a form of adaptation following lower-limb amputation, the potential exists that some of the lumbopelvic and lower-limb movement asymmetries could be "maladaptive," potentially predisposing this population to musculoskeletal disorders such as low back pain (LBP) and osteoarthritis [12-13].

LBP is a common musculoskeletal condition following lower-limb amputation [14-15]. Evidence from prevalence studies confirm that LBP is reported as "more bothersome" than other comorbid conditions such as phantom limb pain and osteoarthritis [16-17]. Further, a majority of respondents with LBP consistently report the presence of LBP for more than $3 \mathrm{yr}$, which suggests the chronic ongoing nature of LBP in this population [15]. ${ }^{*}$ Potential biomechanical contributing factors for ongoing LBP in this population include proximal movement asymmetries at the trunk and lumbopelvic segments secondary to walking with a prosthesis [12]. Increased lumbar transverse rotation has been reported during walking in persons with TFA and LBP as compared with persons with TFA without LBP $(p<0.05$, effect size 1.03) [18]. Despite the cross-sectional study design, the result provides initial evidence for proximal movement asymmetries associated with LBP in people with lower-limb amputation. Such movement and muscle asymmetries in the trunk and lumbopelvic segments could lead to fatigue of spinal musculature and/or cumulative stress of osteoligamentous structures, potentially resulting in spinal instability and LBP $[12,19]$.

Previous systematic reviews have focused mainly on the spatiotemporal parameters and kinetic variables of lower-limb joints during walking [4,20]. Soares et al. reviewed the biomechanical parameters in persons with TTA; however, firm conclusions could not be drawn from this literature review due to the lack of quality assessment of the included studies [20]. Recently, Sagawa et al. reviewed the interlimb movement asymmetries of persons with TFA during stance phase of walking and reported decreased hip motion at the amputated limb in the sagittal plane as compared with the intact limb $(p<$ 0.05 ); nevertheless, the aim of the review was not specific to kinematics because it investigated various biomechanical parameters such as spatiotemporal parameters,

*Devan H, Ribeiro D, Carman A, Hendrick P, Hale L. Functional activity and low back pain in people with lower limb amputation: A national survey. Disabil Rehabil. In review. kinetics, and electromyography [4]. Further, the review included studies conducted on participants with both unilateral and bilateral amputation [4]. A recent review reported the muscle compensatory strategies of persons with TFA and TTA during walking [3]; however, it solely investigated kinetic variables such as joint moment, power, and work of lower-limb joints.

Proximal movement asymmetries at the trunk and lumbopelvic segments during walking have received less attention in the lower-limb amputation literature. In addition to walking, it is equally important to investigate other daily tasks such as climbing stairs, walking uphill and downhill, and running. Understanding the proximal movement asymmetries at the trunk and lumbopelvic segments will inform future prospective studies specifically investigating the potential causal relationship between those movement asymmetries and musculoskeletal disorders such as LBP in this population. The aim of this review is to describe the trunk, lumbopelvic, and hip joint movement asymmetries of the amputated limb during functional tasks as compared with the intact and/or referent limbs of people with TFA and TTA.

\section{METHODS}

\section{Eligibility Criteria}

Our review was limited to observational studies, including cross-sectional, case series, and case studies, because they provide background information for future case-control and prospective studies [21]. Studies involving adults with unilateral TFA and TTA due to all causes of amputation were included. Participants had to be independent while performing functional tasks, which included every day activities such as, but not limited to, walking, stair climbing, lifting or bending, sit-to-stand, and running. The main outcome variable included kinematics of trunk, lumbopelvic, and/or hip joint during functional tasks. For the purpose of the review, asymmetry is defined as a statistically significant difference between the amputated limb and the intact limb and/or the joint segments of nondisabled controls [3-4]. For trunk and lumbopelvic segments, studies comparing amputated and intact sides and persons with amputation and nondisabled controls were included. For hip joint, studies comparing amputated and intact legs and amputated and referent legs of nondisabled controls were included [3]. Studies solely investigating postural control and physiological parameters of participants and 
comparing different prosthetic foot components during functional tasks were excluded. Peer-reviewed articles published in languages other than English and conference proceedings without full text were excluded.

\section{Literature Search}

A comprehensive search strategy was devised (Appendix, available online only) in consultation with a liaison librarian, including the key words "amputation," "adaptation," "asymmetry," "compensation," and "kinematics." The following databases were searched: MEDLINE (via Ovid), EMBASE, AMED (via Ovid), PsycINFO (via Ovid), Cochrane Library (via Ovid), PubMed, CINAHL, Academic Search Complete, SPORTDiscus (via EBSCO), Scopus, Science Direct, Web of Science, Google Scholar, and ProQuest (conference papers and proceedings) from inception to week 3 of February 2014. The primary investigator (H.D.) conducted a hand search of references from the included studies and previous systematic reviews [3$4,20]$. The primary investigator also created electronic alerts for the search strategy in major databases such as PubMed, CINAHL, Scopus, and Web of Science to identify potential articles published until March 2014.

\section{Study Selection}

All the references from electronic databases were exported to Endnote X5 (Thomson Reuters; Philadelphia, Pennsylvania). Two reviewers (H.D. and P.S.) independently searched the electronic databases. Following duplicates exclusion, both reviewers independently screened the titles and abstracts for relevancy. Next, full-text articles were screened for potential inclusion. Throughout the process, a third reviewer (A.C.) was available to settle any disagreement between the reviewers.

\section{Risk of Bias in Individual Studies}

The included articles were assessed for methodological quality based on the modified Down and Black quality assessment tool (Table 1) [22]. This assessment tool was chosen due to its high interrater $(r=0.75)$ and test-retest $(r=0.88)$ reliability [22]. Because our review primarily investigated laboratory-based biomechanical studies, items $8,9,14,17,19,21,24$, and 26 in the scale were removed because they are specific to randomized controlled trials. The modified tool had 19 items. Items 13 and 23 were modified, and the term "interventions" was replaced with "functional tasks." Item 4 was modified into "Are the methods clearly described?" Based on previous research, the percentage of total quality scores was classified as high $(>75 \%)$, moderate $(50 \%-74 \%)$, and low $(<50 \%)$ quality [23]. Two reviewers (H.D. and P.S.) independently assessed the quality of included articles; any disagreement was resolved by mutual discussion and a third reviewer (A.C.) was available to resolve any disagreements.

\section{Data Collection Process}

The following information was extracted based on a standardized form of the Cochrane Collaboration of Systematic reviews [24] by the primary investigator (H.D.) and verified by second reviewer (P.S.): study design, functional task, participant characteristics (age, cause and years since amputation, and type of prosthesis), instrumentation, and outcome measures. For trunk and lumbopelvic joint segments, both total motion from the segment and the data from the amputated and intact sides of a particular segment were extracted. For hip joint, total range of motion from the amputated and intact legs of persons with lower-limb amputation were extracted. The trunk motion was defined as movements occurring only at the thoracic segment including both upper and lower thoracic segments [25]. Only the kinematic data at comfortable walking speed and with a neutral prosthetic alignment were extracted, because walking speed could influence the joint kinematics [26].

\section{Synthesis of Results}

The mean difference and 95 percent confidence intervals of kinematic data between the intact and amputated limbs and/or referent limbs of nondisabled controls from individual studies was calculated using Reference Manager 5.2 (Thomson Reuters). For studies without such information, the primary investigator (H.D.) extracted the data from graphs, and where necessary, the authors were contacted via email for additional information. Due to the age of the included articles, this was not always possible. Since we observed considerable variations in presenting the results of kinematic data and limited studies investigating a functional task, pooling study results was not possible. Thus, a descriptive summary of results is presented. The Cochrane Back Review Group rating scale was modified to summarize the level of evidence as strong (consistent findings from multiple high-quality studies), moderate (consistent findings from one high-quality study and one or more moderate- to low-quality studies or multiple moderate- to low-quality studies), limited or conflicting 
JRRD, Volume 52, Number 1, 2015

Table 1.

Modified Down and Black quality assessment scale items.

\begin{tabular}{|c|c|c|}
\hline Reporting (8 items) & Scoring Criteria & Score \\
\hline $\begin{array}{l}\text { 1. Is the hypothesis, purpose, and/or objective of the } \\
\text { study clearly described? }\end{array}$ & Yes/No & Yes $=1$, No $=0$ \\
\hline $\begin{array}{l}\text { 2. Are the main outcomes to be measured clearly } \\
\text { described in the "Introduction" or "Methods" sections? }\end{array}$ & $\begin{array}{l}\text { If the main outcomes are first mentioned in the } \\
\text { "Results" section, the question should be answered } \\
\text { "no." }\end{array}$ & Yes $=1$, No $=0$ \\
\hline $\begin{array}{l}\text { 3. Are the patient characteristics included in the } \\
\text { study clearly described? }\end{array}$ & $\begin{array}{l}\text { Clear description of inclusion and exclusion criteria. } \\
\text { (Studies should specify whether the participants } \\
\text { were healthy with no pathology, illness, or injury } \\
\text { affecting their functional task.) }\end{array}$ & Yes $=1$, No or Unclear $=0$ \\
\hline 4. Are the methods clearly described? & $\begin{array}{l}\text { A. Equipment: } \\
\text { 1. Make. } \\
\text { 2. Model. } \\
\text { 3. Manufacturer. } \\
\text { B. Data collection: } \\
\text { 4. Sample rate. } \\
\text { 5. Number of cameras. } \\
\text { C. Procedure: } \\
\text { 1. Instructions given. } \\
\text { 2. Familiarization/practice. } \\
\text { 3. Placement of markers. } \\
\text { D. Data processing: } \\
\text { 4. Smoothing/filtering. } \\
\text { 5. Normalization. }\end{array}$ & $\begin{array}{l}4 \mathrm{~A}+4 \mathrm{~B}=0.5 \text { mark: Adequate }(\geq 3 / 5)=0.5, \text { No or } \\
\text { Inadequate }=0 ; 4 \mathrm{C}+4 \mathrm{D}=0.5 \text { mark: Adequate } \\
(\geq 3 / 5)=0.5, \text { No or Inadequate }=0 .\end{array}$ \\
\hline
\end{tabular}

5. Are the distributions of principal confounders in each group of subjects to be compared clearly described?

Principal confounding factors:

1. Age, height, weight.

2. Cause of amputation.

3. Years since amputation.

4. Limb-length discrepancy.

5. Residual limb problems and other comorbidities.

6. Side of amputation.

7. Level of amputation.

8. Type of prosthesis.

6. Are the main findings and/or results of the study clearly described?

Tables and graphs should be clearly presented so that the reader can check the major analyses and conclusions.

7. Does the study provide estimates of the random variability in the data for the main outcomes?

Normal distribution: SD, SE, or 95\% CI. Non-normal distribution: interquartile range. If the distribution of the data was not described, it must be assumed that the estimates used were appropriate and the question should be answered "yes."

10. Have actual probability values been reported for the main outcomes except where the probability value is less than 0.001 ?

Actual $p$-value (e.g., $p=0.035$ rather than $p<$ Adequate $(\geq 4 / 8)=1$, No or Inadequate $=0$. 0.05 ).

Yes $=1$, No $=0$

Yes $=1$, No $=0$.

Yes $=1$, No $=0$.

\section{External Validity (3 items)}

11. Were the subjects asked to participate in the study representative of the entire population from which they were recruited?

12. Were those subjects who were prepared to participate representative of the entire population from which they were recruited?

\section{Scoring Criteria}

The study must describe how the participants were recruited. Study would be representative if they comprised the entire source population, an unselected sample of consecutive patients, or a random sample. When a study does not report the proportion of the source population from which the sample is chosen then the question should be marked as "unable to determine."

The proportion of those who agreed should be stated. Validation that the sample was representative would include demonstrating that the distribution of the main confounding factors was the same in the study sample and the source population.
Yes $=1$, No $=0$, Unable to Determine $=0$. 
Table 1. (cont)

Modified Down and Black quality assessment scale items.

\begin{tabular}{|c|c|c|}
\hline External Validity (3 items) & Scoring Criteria & Score \\
\hline $\begin{array}{l}\text { 13. Were the physical tasks investigated representa- } \\
\text { tive of daily functional tasks? }\end{array}$ & $\begin{array}{l}\text { The study should represent some measures to emu- } \\
\text { late the task analyzed being generalizable to daily } \\
\text { functional tasks. For example, self-selected pace of } \\
\text { walking or use of standardized stair length and width. }\end{array}$ & Yes $=1$, No $=0$, Unable to Determine $=0$ \\
\hline
\end{tabular}

$\frac{\text { Internal Validity (Bias) (4 items) }}{15 . \text { Was an attempt made to blind those measuring }}$ the main outcomes of the study?

16. If any of the results of the study were based on "data dredging," was this made clear?

18. Were the statistical tests used to assess the main outcomes appropriate?

20. Were the main outcome measures used accurate (valid and reliable)?

\begin{tabular}{c}
\hline Internal Validity-Confounding (Selection Bias) \\
(3 items)
\end{tabular}

22. Were cohorts of participants in study group and control group recruited at the same time?

23. Were study subjects randomized to different functional tasks?

25. Was there adequate adjustment for confounding in the analyses from which the main findings were drawn?

\section{Scoring Criteria}

Any analysis that had not been planned at the outset of the study should be clearly indicated. If no retrospective unplanned subgroup analyses were reported, then mark "yes."

The statistical techniques used must be appropriate to the data.

For studies where the outcome measures are clearly described, and studies which refer to other biomechanical validation studies or other 3D models, the question should be answered "yes."

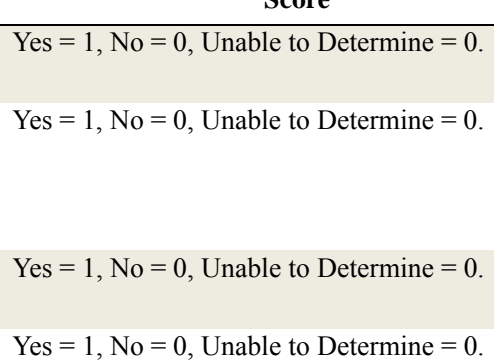

In studies with a single functional task, the scoring $\quad$ Yes $=1$, No $=0$, Not Applicable $=$ NA.

Power (1 item)

$$
\text { Yes }=1, \text { No }=0, \text { Unable to Determine }=0
$$

Yes $=1$, No $=0$, Unable to Determine $=0$.

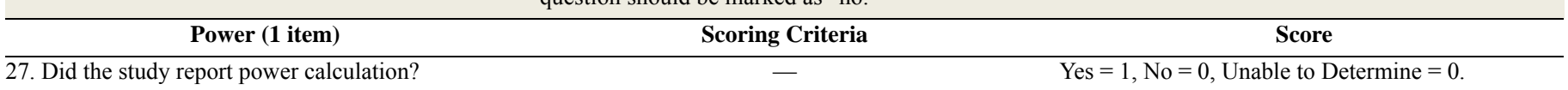

Total: 19 items, 19 marks.

3D $=$ three-dimensional, $\mathrm{CI}=$ confidence interval, $\mathrm{SD}=$ standard deviation, $\mathrm{SE}=$ standard error.

evidence (findings from one high-, moderate-, or lowquality study or inconsistent findings from multiple studies), and no evidence (no studies) [27].

\section{RESULTS}

\section{Study Selection}

The electronic search strategy resulted in 2,679 articles (Figure). After title, abstract, and full-text screening, 21 articles were included for the final review [6-7,9,2845]. Five additional articles were included by searching the references of included articles $(n=3)[8,18,46]$ and from our recent electronic search in February $2014(n=$ 2) [47-48]. No additional articles satisfying the inclusion criteria were retrieved from the electronic alerts. Of the final 26 articles, 9 investigated kinematics in persons with TFA [8-9,18,29-30,35,38-40] and 12 in persons with TTA [6,28,31-34,36,41-42,45-47]; 5 investigated both persons with TFA and TTA $[7,37,43-44,48]$.

\section{Study Characteristics}

\section{Participants}

Table 2 and $\mathbf{3}$ present summaries of included studies investigating persons with TFA and TTA, respectively. Most of the included studies $(n=23)$ adopted a crosssectional study design, with the exception of three studies that had a case series design [7,9,30]. Most of the included studies $(n=17)$ recruited a minimum of 10 participants for the study $[8-9,18,28,31-35,37,40-43,46-$ 48]. The majority (82\%) of amputations were due to either trauma or tumors. 


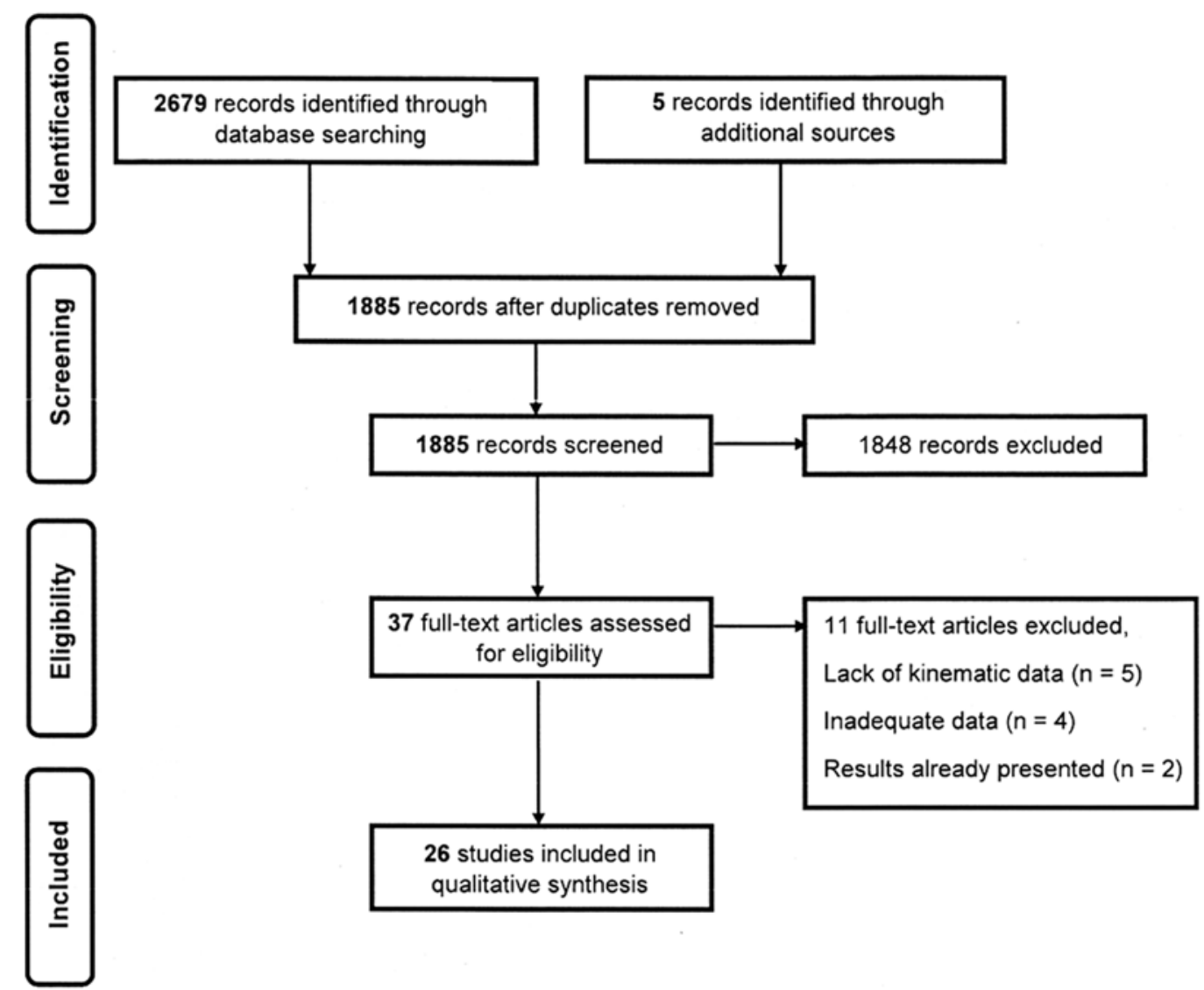

Figure.

Search results.

\section{Outcome Variables}

Overall, 16 studies investigated kinematics during walking [6-9,18,29,32-33,35,38-41,45,47-48] and 4 studies investigated kinematics of stair climbing [28,34,37,42], with few studies investigating the kinematics of other functional tasks, i.e., obstacle crossing $(n=2)[43,46]$, ramp walking $(n=2)[31,44]$, and running $(n=2)[30,36]$. During walking, overall 11 studies investigated pelvis $(n=$ 7) $[7-8,29,35,38-40]$, lumbar spine $(n=1)$ [18], and trunk $(n=4)[8-9,40,48]$ kinematics in participants with TFA. Only three studies investigated pelvis $(n=1)$ [7] and trunk $(n=2)$ [47-48] kinematics in participants with TTA. For functional tasks such as stair climbing, ramp walking, obstacle crossing, and running, only lower-limb kinematics at the hip, knee, and ankle joints were investigated.

\section{Risk of Bias Within Studies}

Table 4 presents the scores of included studies. Most of the studies $(n=20)$ were classified as moderate quality [6-
9,28-34,36-39,44-48], with four classified as high quality $[19,41-43]$ and two classified as low quality $[35,40]$. The total quality assessment score was $12.0 \pm 2.8$ (mean \pm standard deviation). The percentage agreement scores between the two reviewers were good (Cohen kappa: 0.67). Only four studies scored $>50$ percent in both external validity and internal validity sections $[18,33,41,43]$. None of the included studies reported power calculation and all scored poorly for this question.

\section{Results of Studies in Persons with Transfemoral Amputation}

\section{Trunk and Lumbopelvic Kinematics During Walking}

Table 5 presents the results of individual studies and a summary of evidence.

Trunk kinematics. Four studies investigated the trunk motion in participants with TFA $[8-9,40,48]$. Three studies investigated the side-to-side differences in persons with 
Table 2.

Summary of included studies assessing persons with transfemoral amputation (TFA).

\begin{tabular}{|c|c|c|c|c|c|c|c|c|c|c|c|c|c|c|}
\hline \multirow[b]{2}{*}{ Study } & \multirow[b]{2}{*}{ Design } & \multirow[b]{2}{*}{ Activity } & \multicolumn{8}{|c|}{ Participants } & \multirow[b]{2}{*}{ Instrumentation } & \multicolumn{3}{|c|}{ Outcome Measures } \\
\hline & & & \multicolumn{2}{|c|}{ No. } & \multicolumn{2}{|c|}{$\begin{array}{c}\text { Age, yr } \\
\text { (mean } \pm \text { SD } \\
\text { or range) }\end{array}$} & $\begin{array}{c}\text { Cause of } \\
\text { Amputation } \\
\text { I }\end{array}$ & $\begin{array}{c}\text { Time Since } \\
\text { Amputation, } \\
\text { yr (mean } \pm \\
\text { SD) }\end{array}$ & \multicolumn{2}{|c|}{ Prosthesis Type } & & Joint & Comparison & Plane \\
\hline $\begin{array}{l}\text { Bae et al., } \\
2007 \text { [29] }\end{array}$ & $\begin{array}{l}\text { Cross- } \\
\text { sectional }\end{array}$ & Walking & 8 & 10 & $40 \pm 8$ & $24 \pm 2$ & NA & NA & NA & NA & $\begin{array}{l}\text { 7-camera Vicon } \\
\text { (30 markers, } \\
3 \text { DOF) }\end{array}$ & $\begin{array}{l}\text { Pelvis, hip, } \\
\text { knee }\end{array}$ & $\begin{array}{l}\text { INT vs } \\
\text { CON }\end{array}$ & $\begin{array}{l}\text { Sagittal, } \\
\text { frontal }\end{array}$ \\
\hline $\begin{array}{l}\text { Goujon- } \\
\text { Pillet et al., } \\
2008[8]\end{array}$ & $\begin{array}{l}\text { Cross- } \\
\text { sectional }\end{array}$ & Walking & 27 & 33 & 51 & 44 & $\begin{array}{l}\text { Trauma (24), } \\
\text { tumor (2), } \\
\text { congenital (1) }\end{array}$ & $27 \pm 17$ & $\begin{array}{l}\text { Monoaxial } \\
\text { (19), poly- } \\
\text { centric (8) }\end{array}$ & - & $\begin{array}{l}\text { 12-camera } \\
\text { Vicon } \\
\text { (46 markers, } \\
6 \text { DOF) }\end{array}$ & $\begin{array}{l}\text { Trunk, } \\
\text { pelvis }\end{array}$ & $\begin{array}{l}\text { AMP vs } \\
\text { CON }\end{array}$ & $\begin{array}{l}\text { Sagittal, } \\
\text { frontal, } \\
\text { horizontal }\end{array}$ \\
\hline $\begin{array}{l}\text { Jaegers et al., } \\
1995[9]\end{array}$ & $\begin{array}{l}\text { Case } \\
\text { series }\end{array}$ & Walking & 11 & 2 & 36 & $\mathrm{NA}$ & $\begin{array}{l}\text { Trauma or } \\
\text { tumor }\end{array}$ & NA & $\begin{array}{l}\text { Monoaxial } \\
\text { (3), polycen- } \\
\text { tric ( } 8 \text { ) }\end{array}$ & Multiflex & $\begin{array}{l}\text { Electrogoniome- } \\
\text { ters (trunk, hip, } \\
\text { and knee angles) }\end{array}$ & $\begin{array}{l}\text { Trunk, hip, } \\
\text { knee }\end{array}$ & $\begin{array}{l}\text { AMP vs } \\
\text { INT }\end{array}$ & $\begin{array}{l}\text { Sagittal, } \\
\text { frontal }\end{array}$ \\
\hline $\begin{array}{l}\text { Michaud } \\
\text { et al., } 2000 \\
\text { [7] }\end{array}$ & $\begin{array}{l}\text { Case } \\
\text { series }\end{array}$ & Walking & 3 & - & $52 \pm 18$ & - & $\begin{array}{l}\text { Trauma or } \\
\text { tumor }\end{array}$ & NA & Hydraulic & $\begin{array}{l}\text { Flexfoot (2), } \\
\text { Seattle foot } \\
\text { (1) }\end{array}$ & $\begin{array}{l}\text { CODA } 3 \text { sys- } \\
\text { tem }(2 \text { markers, } \\
1 \text { DOF })\end{array}$ & Pelvis & $\begin{array}{l}\text { AMP vs } \\
\text { INT }\end{array}$ & Frontal \\
\hline $\begin{array}{l}\text { Morgenroth } \\
\text { et al., } 2010 \\
{[18]}\end{array}$ & $\begin{array}{l}\text { Cross- } \\
\text { sectional }\end{array}$ & Walking & 17 & 6 & 49 & $32 \pm 8$ & $\begin{array}{l}\text { Trauma (14), } \\
\text { tumor (1), } \\
\text { congenital (1), } \\
\text { vascular (1) }\end{array}$ & NA & NA & NA & $\begin{array}{l}\text { 10-camera } \\
\text { Vicon } \\
\text { (41 markers, } \\
6 \text { DOF) }\end{array}$ & $\begin{array}{l}\text { Lumbar } \\
\text { spine }\end{array}$ & $\begin{array}{l}\text { AMP vs } \\
\text { CON }\end{array}$ & $\begin{array}{l}\text { Sagittal, } \\
\text { frontal, } \\
\text { horizontal }\end{array}$ \\
\hline $\begin{array}{l}\text { Rabuffetti } \\
\text { et al., } 2005 \\
\text { [35] }\end{array}$ & $\begin{array}{l}\text { Cross- } \\
\text { sectional }\end{array}$ & Walking & 14 & 7 & $19-54$ & $24-51$ & NA & NA & NA & NA & $\begin{array}{l}\text { 4-camera system } \\
\text { (12 markers, } 3 \\
\text { DOF) }\end{array}$ & Pelvis, hip & $\begin{array}{l}\text { AMP vs } \\
\text { INT }\end{array}$ & Sagittal \\
\hline $\begin{array}{l}\text { Sjödahl } \\
\text { et al., 2003 } \\
\text { [39] }\end{array}$ & $\begin{array}{l}\text { Cross- } \\
\text { sectional }\end{array}$ & Walking & 9 & 18 & 33 & 36 & $\begin{array}{l}\text { Trauma or } \\
\text { tumor }\end{array}$ & $10 \pm 6$ & $\begin{array}{l}\text { Pneumatic } \\
\text { (6), mechani- } \\
\text { cal (3), } \\
\text { hydraulic (2) }\end{array}$ & $\begin{array}{l}\text { Flexfoot } \\
(6), \text { Seattle } \\
\text { foot (2), } \\
\text { multiflex } \\
\text { ankle (1) }\end{array}$ & $\begin{array}{l}\text { 5-camera Vicon } \\
\text { (21 markers, } \\
3 \text { DOF) }\end{array}$ & $\begin{array}{l}\text { Pelvis, hip, } \\
\text { knee }\end{array}$ & $\begin{array}{l}\text { AMP vs } \\
\text { INT, AMP } \\
\text { vs CON }\end{array}$ & $\begin{array}{l}\text { Frontal, } \\
\text { horizontal }\end{array}$ \\
\hline $\begin{array}{l}\text { Tazawa, } \\
1997[40]\end{array}$ & $\begin{array}{l}\text { Cross- } \\
\text { sectional }\end{array}$ & Walking & 12 & - & NA & - & NA & NA & NA & NA & $\begin{array}{l}\text { 6-camera Vicon } \\
\text { (12 markers, } \\
6 \text { DOF) }\end{array}$ & Pelvis, trunk & $\begin{array}{l}\text { AMP vs } \\
\text { INT }\end{array}$ & $\begin{array}{l}\text { Sagittal, } \\
\text { frontal, } \\
\text { horizontal }\end{array}$ \\
\hline $\begin{array}{l}\text { Vrieling et } \\
\text { al., } 2007 \text { [43] }\end{array}$ & $\begin{array}{l}\text { Cross- } \\
\text { sectional }\end{array}$ & $\begin{array}{l}\text { Obstacle } \\
\text { crossing }\end{array}$ & 8 & 10 & $46 \pm 15$ & $45 \pm 9$ & $\begin{array}{l}\text { Trauma (4), } \\
\text { tumor (3), } \\
\text { vascular (1) }\end{array}$ & $\mathrm{U}$ & NA & NA & $\begin{array}{l}\text { Electrogoniome- } \\
\text { ters (hip, knee, } \\
\text { and ankle joints) }\end{array}$ & $\begin{array}{l}\text { Hip, knee, } \\
\text { ankle }\end{array}$ & $\begin{array}{l}\text { AM P vs } \\
\text { INT, AMP } \\
\text { vs CON }\end{array}$ & Sagittal \\
\hline $\begin{array}{l}\text { Vrieling et } \\
\text { al., } 2008 \text { [44] }\end{array}$ & $\begin{array}{l}\text { Cross- } \\
\text { sectional }\end{array}$ & $\begin{array}{l}\text { Ramp } \\
\text { walking }\end{array}$ & 7 & 10 & 44 & 45 & $\begin{array}{l}\text { Trauma (4), } \\
\text { tumor (3) }\end{array}$ & $\mathrm{U}$ & $\begin{array}{l}\text { Graph-lite } \\
\text { (3), C-Leg } \\
\text { (1),3R60(1), } \\
\text { Safelife (1), } \\
\text { Total Knee } \\
\text { (1) }\end{array}$ & $\begin{array}{l}\text { Multiflex } \\
\text { (3), SACH } \\
\text { (2), C-Walk } \\
\text { (1) }\end{array}$ & $\begin{array}{l}\text { Electrogoniome- } \\
\text { ters (hip, knee, } \\
\text { and ankle joints) }\end{array}$ & $\begin{array}{l}\text { Hip, knee, } \\
\text { ankle }\end{array}$ & $\begin{array}{l}\text { AM P vs } \\
\text { INT, AMP } \\
\text { vs CON }\end{array}$ & Sagittal \\
\hline
\end{tabular}

$\overline{\mathrm{AMP}}=$ amputated limb, $\mathrm{CON}=$ nondisabled control, $\mathrm{DOF}=$ degrees of freedom, INT $=$ intact limb of person with amputation, $\mathrm{NA}=$ not available, $\mathrm{SACH}=$ solid ankle cushion heel, $\mathrm{SD}=$ standard deviation, $\mathrm{U}=$ unknown. 
JRRD, Volume 52, Number 1, 2015

Table 3.

Summary of included studies assessing persons with transtibial amputation (TTA).

\begin{tabular}{|c|c|c|c|c|c|c|c|c|c|c|c|c|c|}
\hline \multirow{3}{*}{ Study } & \multirow{3}{*}{ Design } & \multirow{3}{*}{ Activity } & \multicolumn{7}{|c|}{ Participants } & \multirow{3}{*}{ Instrumentation } & \multicolumn{3}{|c|}{ Outcome Measures } \\
\hline & & & \multicolumn{2}{|c|}{ No. } & \multicolumn{2}{|c|}{$\begin{array}{c}\text { Age, yr } \\
(\text { mean } \pm \text { SD) }\end{array}$} & \multirow{2}{*}{$\begin{array}{c}\text { Cause of } \\
\text { Amputation }\end{array}$} & \multirow{2}{*}{$\begin{array}{c}\text { Time Since } \\
\text { Amputation, } \\
\text { yr (mean } \pm \\
\text { SD) }\end{array}$} & \multirow{2}{*}{$\begin{array}{l}\text { Prosthesis } \\
\text { Foot Type }\end{array}$} & & \multirow{2}{*}{ Joint } & \multirow{2}{*}{ Comparison } & \multirow{2}{*}{ Plane } \\
\hline & & & TTA & Control & TTA & Control & & & & & & & \\
\hline $\begin{array}{l}\text { Alimusaj et } \\
\text { al., } 2009 \text { [28] }\end{array}$ & $\begin{array}{l}\text { Cross- } \\
\text { sectional }\end{array}$ & $\begin{array}{l}\text { Stair } \\
\text { climbing }\end{array}$ & 16 & 16 & $50 \pm 12$ & $31 \pm 10$ & $\begin{array}{l}\text { Trauma (13), } \\
\text { tumor (3) }\end{array}$ & $25 \pm 21$ & ProprioFoot & $\begin{array}{l}\text { Vicon (number of } \\
\text { cameras and mark- } \\
\text { ers =NA, } 6 \text { DOF) }\end{array}$ & $\begin{array}{l}\text { Hip, knee, } \\
\text { ankle }\end{array}$ & $\begin{array}{l}\text { AMP vs } \\
\text { CON }\end{array}$ & Sagittal \\
\hline $\begin{array}{l}\text { Fradet et al., } \\
2010[31]\end{array}$ & $\begin{array}{l}\text { Cross- } \\
\text { sectional }\end{array}$ & $\begin{array}{l}\text { Ramp } \\
\text { walking }\end{array}$ & 16 & 16 & $50 \pm 12$ & $31 \pm 10$ & $\begin{array}{l}\text { Trauma (13), } \\
\text { tumor (3) }\end{array}$ & $25 \pm 21$ & ProprioFoot & $\begin{array}{l}\text { Vicon (number of } \\
\text { cameras and mark- } \\
\text { ers =NA, } 6 \text { DOF) }\end{array}$ & $\begin{array}{l}\text { Hip, knee, } \\
\text { ankle }\end{array}$ & $\begin{array}{l}\text { AMP vs } \\
\text { CON }\end{array}$ & Sagittal \\
\hline $\begin{array}{l}\text { Gates et al., } \\
2012 \text { [32] }\end{array}$ & $\begin{array}{l}\text { Cross- } \\
\text { sectional }\end{array}$ & Walking & 13 & 11 & $28 \pm 4$ & NA & Trauma & NA & $\begin{array}{l}\text { Monoaxial energy } \\
\text { storing }\end{array}$ & $\begin{array}{l}\text { 20-camera system } \\
(55 \text { markers, } \\
6 \text { DOF })\end{array}$ & $\begin{array}{l}\text { Hip, knee, } \\
\text { ankle }\end{array}$ & $\begin{array}{l}\text { AMP vs } \\
\text { CON, AMP } \\
\text { vs INT }\end{array}$ & Sagittal \\
\hline $\begin{array}{l}\text { Hill et al., } \\
1997 \text { [46] }\end{array}$ & $\begin{array}{l}\text { Cross- } \\
\text { sectional }\end{array}$ & $\begin{array}{l}\text { Obstacle } \\
\text { crossing }\end{array}$ & 10 & - & $38 \pm 5$ & - & Trauma & $2 \pm 0.4$ & $\begin{array}{l}\text { Flexfoot (4), } \\
\text { Re-Flex Pylon (3), } \\
\text { Seattle foot (2), } \\
\text { Safe foot (1) }\end{array}$ & $\begin{array}{l}\text { 5-camera Vicon } \\
\text { (14 markers, } \\
3 \text { DOF) }\end{array}$ & $\begin{array}{l}\text { Trunk, hip, } \\
\text { knee, ankle }\end{array}$ & AMP vs INT & Sagittal \\
\hline $\begin{array}{l}\text { Kovač et al., } \\
2010[33]\end{array}$ & $\begin{array}{l}\text { Cross- } \\
\text { sectional }\end{array}$ & Walking & 12 & 12 & $40 \pm 6$ & $37 \pm 5$ & Trauma & $10 \pm 1$ & $\begin{array}{l}\text { Dynamic foot (7), } \\
\text { Greissenger (2), } \\
\text { Flexfoot (2) }\end{array}$ & $\begin{array}{l}\text { 8-camera system } \\
\text { (markers = NA, } \\
6 \text { DOF) }\end{array}$ & $\begin{array}{l}\text { Hip, knee, } \\
\text { ankle }\end{array}$ & AMP vs INT & $\begin{array}{l}\text { Sagittal, } \\
\text { frontal }\end{array}$ \\
\hline $\begin{array}{l}\text { Michaud et } \\
\text { al., } 2000 \text { [7] }\end{array}$ & $\begin{array}{l}\text { Case } \\
\text { series }\end{array}$ & Walking & 6 & - & $41 \pm 13$ & - & $\begin{array}{l}\text { Trauma or } \\
\text { tumor }\end{array}$ & NA & $\begin{array}{l}\text { Flexfoot (2), } \\
\text { Greissenger (1), } \\
\text { Seattle foot (1), } \\
\text { Carbon Copy II (1) }\end{array}$ & $\begin{array}{l}\text { CODA } 3 \text { system } \\
\text { (2 markers, } 1 \text { DOF) }\end{array}$ & Pelvis & AMP vs INT & Frontal \\
\hline $\begin{array}{l}\text { Powers et al., } \\
1997 \text { [34] }\end{array}$ & $\begin{array}{l}\text { Cross- } \\
\text { sectional }\end{array}$ & $\begin{array}{l}\text { Stair } \\
\text { climbing }\end{array}$ & 10 & 14 & $51 \pm 15$ & $34 \pm 11$ & $\begin{array}{l}\text { Trauma (8), } \\
\text { vascular (2) }\end{array}$ & $15 \pm 15$ & Seattle Light Foot & $\begin{array}{l}\text { 6-camera Vicon } \\
(23 \text { markers, } \\
6 \text { DOF) }\end{array}$ & $\begin{array}{l}\text { Pelvis, hip, } \\
\text { knee, ankle }\end{array}$ & $\begin{array}{l}\text { AMP vs } \\
\text { CON }\end{array}$ & Sagittal \\
\hline $\begin{array}{l}\text { Molina Rueda } \\
\text { et al., } 2013 \\
{[47]}\end{array}$ & $\begin{array}{l}\text { Cross- } \\
\text { sectional }\end{array}$ & Walking & 15 & 15 & $56 \pm 14$ & $48 \pm 14$ & $\begin{array}{l}\text { Trauma (12), } \\
\text { tumor (3) }\end{array}$ & NA & $\begin{array}{l}\text { Flexfoot (7), } \\
\text { Ceterus (2), } \\
\text { Variflex (2), SACH } \\
\text { (1), Talux (1), Trias } \\
\text { (1), Quantum (1) }\end{array}$ & $\begin{array}{l}\text { 8-camera Vicon } \\
\text { (23 markers, } \\
6 \text { DOF) }\end{array}$ & Pelvis, trunk & $\begin{array}{l}\text { AMP vs } \\
\text { CON }\end{array}$ & Frontal \\
\hline $\begin{array}{l}\text { Sanderson \& } \\
\text { Martin, } 1996 \\
{[36]}\end{array}$ & $\begin{array}{l}\text { Cross- } \\
\text { sectional }\end{array}$ & Running & 6 & 6 & $40 \pm 7$ & $33 \pm 7$ & NA & $12 \pm 9$ & Flexfoot & $\begin{array}{l}\text { Single video cam- } \\
\text { era (markers = NA, } \\
3 \text { DOF) }\end{array}$ & $\begin{array}{l}\text { Hip, knee, } \\
\text { ankle }\end{array}$ & $\begin{array}{l}\text { AMP vs INT, } \\
\text { AMP vs } \\
\text { CON }\end{array}$ & Sagittal \\
\hline $\begin{array}{l}\text { Sanderson \& } \\
\text { Martin, } 1997 \\
{[6]}\end{array}$ & $\begin{array}{l}\text { Cross- } \\
\text { sectional }\end{array}$ & Walking & 6 & 6 & $40 \pm 7$ & $33 \pm 7$ & Trauma & 12 & Flexfoot & $\begin{array}{l}\text { Single video cam- } \\
\text { era ( } 10 \text { markers, } \\
3 \text { DOF) }\end{array}$ & $\begin{array}{l}\text { Hip, knee, } \\
\text { ankle }\end{array}$ & $\begin{array}{l}\text { AMP vs INT, } \\
\text { AMP vs } \\
\text { CON }\end{array}$ & Sagittal \\
\hline $\begin{array}{l}\text { Schmalz et } \\
\text { al., } 2007 \text { [37] }\end{array}$ & $\begin{array}{l}\text { Cross- } \\
\text { sectional }\end{array}$ & $\begin{array}{l}\text { Stair } \\
\text { climbing }\end{array}$ & 8 & 12 & $51 \pm 14$ & $30 \pm 10$ & $\begin{array}{l}\text { Trauma (7), } \\
\text { tumor (1) }\end{array}$ & $27 \pm 17$ & C-Leg & $\begin{array}{l}\text { 4-camera PRIMAS } \\
\text { (8 markers, } 3 \text { DOF) }\end{array}$ & Knee, ankle & AMP vs INT & Sagittal \\
\hline $\begin{array}{l}\text { Vanicek et al., } \\
2009 \text { [41] }\end{array}$ & $\begin{array}{l}\text { Cross- } \\
\text { sectional }\end{array}$ & Walking & 11 & - & $56 \pm 13$ & - & $\begin{array}{l}\text { Trauma (6), } \\
\text { vascular (3), } \\
\text { congenital (2) }\end{array}$ & $7 \pm 7$ & $\begin{array}{l}\text { Multiflex (7), } \\
\text { Variflex (2), } \\
\text { Dynamic (1), } \\
\text { Ceterus (1) }\end{array}$ & $\begin{array}{l}\text { 10-camera Qualy- } \\
\text { sis }(28 \text { markers, } \\
6 \text { DOF })\end{array}$ & $\begin{array}{l}\text { Hip, knee, } \\
\text { ankle }\end{array}$ & $\begin{array}{l}\text { AMP } \\
\text { (fallers) vs } \\
\text { AMP (non- } \\
\text { fallers) }\end{array}$ & $\begin{array}{l}\text { Sagittal, } \\
\text { frontal }\end{array}$ \\
\hline $\begin{array}{l}\text { Vanicek et al., } \\
2010 \text { [42] }\end{array}$ & $\begin{array}{l}\text { Cross- } \\
\text { sectional }\end{array}$ & $\begin{array}{l}\text { Stair } \\
\text { climbing }\end{array}$ & 11 & - & $56 \pm 13$ & - & $\begin{array}{l}\text { Trauma (6), } \\
\text { vascular (3), } \\
\text { congenital (2) }\end{array}$ & $7 \pm 7$ & $\begin{array}{l}\text { Multiflex (7), } \\
\text { Variflex (2), } \\
\text { Dynamic (1), } \\
\text { Ceterus (1) }\end{array}$ & $\begin{array}{l}\text { 10-camera Qualy- } \\
\text { sis }(28 \text { markers, } \\
6 \text { DOF) }\end{array}$ & $\begin{array}{l}\text { Hip, knee, } \\
\text { ankle }\end{array}$ & $\begin{array}{l}\text { AMP } \\
\text { (fallers) vs } \\
\text { AMP (non- } \\
\text { fallers) }\end{array}$ & Sagittal \\
\hline $\begin{array}{l}\text { Vrieling et al., } \\
2007 \text { [43] }\end{array}$ & $\begin{array}{l}\text { Cross- } \\
\text { sectional }\end{array}$ & $\begin{array}{l}\text { Obstacle } \\
\text { crossing }\end{array}$ & 12 & 10 & $50 \pm 12$ & $45 \pm 9$ & $\begin{array}{l}\text { Trauma (6), } \\
\text { tumor (4), } \\
\text { vascular (2) }\end{array}$ & $17 \pm 14$ & NA & $\begin{array}{l}\text { Electrogoniome- } \\
\text { ters (hip, knee, and } \\
\text { ankle joints }\end{array}$ & $\begin{array}{l}\text { Hip, knee, } \\
\text { ankle }\end{array}$ & $\begin{array}{l}\text { AMP vs INT, } \\
\text { AMP vs } \\
\text { CON }\end{array}$ & Sagittal \\
\hline $\begin{array}{l}\text { Yeung et al., } \\
2012[45]\end{array}$ & $\begin{array}{l}\text { Cross- } \\
\text { sectional }\end{array}$ & Walking & 6 & - & $53 \pm 9$ & - & $\begin{array}{l}\text { Trauma (5), } \\
\text { vascular (1) }\end{array}$ & - & $\begin{array}{l}\text { SACH (5), ESAR } \\
\text { (1) }\end{array}$ & $\begin{array}{l}\text { 8-camera Vicon } \\
(24 \text { markers, } \\
6 \text { DOF) }\end{array}$ & $\begin{array}{l}\text { Hip, knee, } \\
\text { ankle }\end{array}$ & AMP vs INT & Sagittal \\
\hline
\end{tabular}

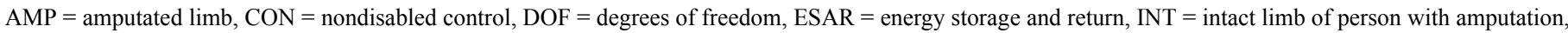
$\mathrm{NA}=$ not available, $\mathrm{SACH}=$ solid ankle cushion heel, $\mathrm{SD}=$ standard deviation. 
Table 4.

Methodological quality of included studies. Quality rating criteria: high $(\geq 75 \%)$, moderate $(50 \%-74 \%)$, and low $(<50 \%)$.

\begin{tabular}{|c|c|c|c|c|c|c|c|c|c|c|c|c|c|c|c|c|c|c|c|c|c|c|c|c|c|}
\hline \multirow{3}{*}{ Study } & \multicolumn{25}{|c|}{ Modified Down and Black Scale Items } \\
\hline & \multicolumn{9}{|c|}{ Reporting } & \multicolumn{4}{|c|}{ External Validity } & \multicolumn{5}{|c|}{$\begin{array}{c}\text { Internal Validity } \\
\text { (Bias) }\end{array}$} & \multicolumn{4}{|c|}{ Confounding } & \multicolumn{2}{|c|}{ Power } & \multirow[t]{2}{*}{ Total } \\
\hline & 1 & 2 & 3 & 4 & 5 & 6 & 7 & 10 & $\%$ & 11 & 12 & 13 & $\%$ & $\overline{15}$ & 16 & 18 & 20 & $\%$ & 22 & 23 & 25 & $\overline{\%}$ & 27 & $\%$ & \\
\hline Bae et al., 2007 [29] & 1 & 1 & 1 & 1 & 1 & 1 & 1 & 0 & 88 & 0 & 0 & 0 & 0 & 0 & 1 & 1 & 1 & 75 & 0 & 0 & 1 & 33 & 0 & 0 & 58 \\
\hline Burkett et al., 2003 [30] & 1 & 1 & 1 & 1 & 1 & 1 & 1 & 0 & 100 & 0 & 0 & 1 & 33 & 0 & 1 & 1 & 1 & 75 & NA & NA & NA & NA & NA & NA & 73 \\
\hline Fradet et al., 2010 [31] & 1 & 1 & 1 & 1 & 1 & 1 & 1 & 0 & 88 & 0 & 0 & 1 & 33 & 0 & 1 & 1 & 1 & 75 & 0 & 0 & 0 & 0 & 0 & 0 & 58 \\
\hline Goujon-Pillet et al., 2008 [8] & 1 & 1 & 1 & 1 & 1 & 1 & 1 & 1 & 100 & 0 & 0 & 1 & 33 & 0 & 1 & 1 & 1 & 75 & 0 & NA & 1 & 50 & 0 & 0 & 72 \\
\hline Hendershot \& Wolf, 2014 [48] & 1 & 1 & 1 & 1 & 1 & 1 & 1 & 1 & 100 & 0 & 0 & 1 & 33 & 0 & 1 & 1 & 1 & 75 & 0 & NA & 1 & 50 & 0 & 0 & 72 \\
\hline Hill et al., 1997 [46] & 1 & 1 & 1 & 1 & 1 & 1 & 0 & 0 & 75 & 0 & 0 & 1 & 33 & 0 & 1 & 1 & 1 & 75 & 0 & 1 & 0 & 33 & 0 & 0 & 58 \\
\hline Jaegers et al., 1995 [9] & 1 & 1 & 1 & 1 & 1 & 1 & 1 & 1 & 100 & 0 & 0 & 1 & 33 & 0 & 1 & 1 & 0 & 50 & 0 & NA & 0 & 0 & NA & NA & 65 \\
\hline Kovač et al., 2010 [33] & 0 & 1 & 1 & 1 & 1 & 0 & 1 & 1 & 75 & 1 & 0 & 1 & 67 & 0 & 0 & 1 & 1 & 50 & 0 & NA & 1 & 50 & 0 & 0 & 61 \\
\hline Molina Rueda et al., 2013 [47] & 1 & 1 & 1 & 1 & 1 & 1 & 1 & 0 & 88 & 0 & 0 & 1 & 33 & 0 & 1 & 1 & 1 & 75 & 0 & NA & 0 & 0 & 0 & 0 & 61 \\
\hline Sanderson \& Martin, 1996 [36] & 1 & 1 & 1 & 1 & 1 & 1 & 1 & NA & 100 & 0 & 0 & 1 & 33 & 0 & 1 & 1 & 1 & 75 & 0 & 1 & 0 & 33 & 0 & 0 & 67 \\
\hline Sanderson \& Martin, 1997 [6] & 1 & 1 & 1 & 1 & 1 & 1 & 1 & 1 & 100 & 0 & 0 & 1 & 33 & 0 & 1 & 1 & 1 & 75 & 0 & 1 & 0 & 33 & 0 & 0 & 68 \\
\hline Schmalz et al., 2007 [37] & 1 & 1 & 1 & 1 & 1 & 1 & 1 & 0 & 88 & 0 & 0 & 0 & 0 & 0 & 1 & 1 & 1 & 75 & 0 & 0 & 0 & 0 & 0 & 0 & 53 \\
\hline Sjödahl et al., 2002 [38] & 1 & 1 & 1 & 1 & 1 & 1 & 0 & 1 & 88 & 0 & 1 & 1 & 67 & 0 & 1 & 1 & 1 & 75 & 0 & NA & 0 & 0 & 0 & 0 & 67 \\
\hline Sjödahl et al., 2003 [39] & 1 & 1 & 1 & 1 & 1 & 1 & 1 & 0 & 88 & 0 & 1 & 1 & 67 & 0 & 1 & 1 & 1 & 75 & 0 & NA & 0 & 0 & 0 & 0 & 67 \\
\hline Tazawa, 1997 [40] & 1 & 1 & 1 & 1 & 0 & 1 & 0 & 0 & 63 & 0 & 0 & 1 & 33 & 0 & 1 & 0 & 0 & 25 & 0 & NA & 0 & 0 & 0 & 0 & 39 \\
\hline Vanicek et al., 2009 [41] & 1 & 1 & 1 & 1 & 1 & 1 & 1 & 1 & 100 & 1 & 0 & 1 & 67 & 0 & 1 & 1 & 1 & 75 & 1 & NA & 1 & 100 & 0 & 0 & 83 \\
\hline Vanicek et al., 2010 [42] & 1 & 1 & 1 & 1 & 1 & 1 & 1 & 1 & 100 & 0 & 0 & 1 & 33 & 0 & 1 & 1 & 1 & 75 & 1 & NA & 1 & 100 & 0 & 0 & 78 \\
\hline Vrieling et al., 2007 [43] & 1 & 1 & 1 & 1 & 1 & 1 & 1 & 1 & 100 & 1 & 0 & 1 & 67 & 0 & 1 & 1 & 1 & 75 & 0 & 1 & 1 & 67 & 0 & 0 & 79 \\
\hline
\end{tabular}

TFA [9,40,48]. During stance phase of the amputated limb, increased trunk lateral flexion toward the amputated limb $(p<0.05)$ was reported as compared with the intact limb. Two studies compared the sagittal and frontal trunk motion among participants with TFA and nondisabled controls and reported increased trunk motion $(p<0.05)$ in sagittal and frontal plane during walking $[8,48]$.

Pelvic kinematics. Frontal plane-Five studies investigated pelvic obliquity during walking [7-8,29,39-40]. Three studies investigated the differences in pelvic obliquity between the intact and prosthetic limbs [7,39-40]. During the loading response phase of walking, the magnitude of pelvic obliquity on the amputated side was smaller than on the intact side $(p>0.05)$ [39]. Further, during single-leg support phase of both limbs, a hip-hiking pattern was observed with the pelvis of the swing limb adopting a more elevated position than the stance limb $(p>0.05)$ [39]. There was a concomitant ipsilateral leaning of the trunk region during this single-leg support phase $[7,39,48]$. Two studies compared the pelvic obliquity among persons with TFA and nondisabled controls and concluded increased total pelvic obliquity $(p<0.05)$ in persons with TFA (Table 6) $[8,29]$. Sagittal plane-Three studies investigated pelvic tilt during walking $[8,35,38]$. Only one study investigated the differences in pelvic tilt between amputated and intact legs specifically during initial contact phase of walking [35]. The results showed a significant increase in anterior pelvic tilt of the amputated limb as compared with the intact limb [35]. Among studies comparing pelvic tilt of participants with TFA and nondisabled controls, two studies reported increased pelvic tilt $(p<0.05)$ as compared with nondisabled controls $[8,38]$. Transverse plane-Three studies 
JRRD, Volume 52, Number 1, 2015

Table 5.

Summary of evidence: During walking for persons with transfemoral amputation.

\begin{tabular}{|c|c|c|c|c|}
\hline Study & Comparison & $\begin{array}{c}\text { Mean Difference } \\
\text { (95\% CI) }\end{array}$ & Findings & $\begin{array}{c}\text { Summary of } \\
\text { Evidence }\end{array}$ \\
\hline \multicolumn{5}{|l|}{ Trunk } \\
\hline \multicolumn{5}{|l|}{ Sagittal Plane } \\
\hline Goujon-Pillet et al., 2008 [8] & AMP vs CON & $3(2-4)$ & $\mathrm{AMP}>\mathrm{CON}$ & Limited \\
\hline Hendershot \& Wolf, 2014 [48] & AMP vs CON & $3.7(2.7-4.7)$ & $\mathrm{AMP}>\mathrm{CON}$ & - \\
\hline \multicolumn{5}{|l|}{ Frontal Plane } \\
\hline Goujon-Pillet et al., 2008 [8] & AMP vs CON & $4(3-5)$ & $\mathrm{AMP}>\mathrm{CON}$ & - \\
\hline Hendershot \& Wolf, 2014 [48] & AMP vs CON & $4(3-5)$ & $\mathrm{AMP}>\mathrm{CON}$ & Moderate \\
\hline Hendershot \& Wolf, 2014 [48] & AMP vs INT & $3.6(2.5-4.7)$ & $\mathrm{AMP}>\mathrm{INT}$ & - \\
\hline Jaegers et al., 1995 [9] & AMP vs INT & $5(1-9)$ & $\mathrm{AMP}>\mathrm{INT}$ & - \\
\hline \multicolumn{5}{|l|}{ Horizontal Plane } \\
\hline Goujon-Pillet et al., 2008 [8] & AMP vs CON & $3(1-5)$ & $\mathrm{AMP}>\mathrm{CON}$ & Limited \\
\hline \multicolumn{5}{|l|}{ Pelvis } \\
\hline \multicolumn{5}{|l|}{ Frontal Plane } \\
\hline Bae et al., 2007 [29] & AMP vs CON & $-5(-12$ to 2$)$ & NS & Limited \\
\hline Goujon-Pillet et al., 2008 [8] & AMP vs CON & $2(0.5-3)$ & $\mathrm{AMP}>\mathrm{CON}$ & - \\
\hline \multicolumn{5}{|l|}{ Sagittal Plane } \\
\hline Rabuffetti et al., 2005 [35] & AMP vs INT & $8.2^{\dagger}$ & $\mathrm{AMP}>\mathrm{INT}$ & Moderate \\
\hline Goujon-Pillet et al., 2008 [8] & AMP vs CON & $4(2-6)$ & $\mathrm{AMP}>\mathrm{CON}$ & - \\
\hline Sjödahl et al., 2002 [38] & AMP vs CON & $8^{\dagger}$ & $\mathrm{AMP}>\mathrm{CON}$ & - \\
\hline \multicolumn{5}{|l|}{ Transverse Plane } \\
\hline Goujon-Pillet et al., 2008 [8] & AMP vs CON & $-0.2(-4$ to 3$)$ & NS & Limited \\
\hline \multicolumn{5}{|l|}{ Lumbar Spine } \\
\hline \multicolumn{5}{|l|}{ Sagittal Plane } \\
\hline Morgenroth et al., 2010 [18] & AMP vs CON & $10(4-15)$ & $\mathrm{AMP}>\mathrm{CON}$ & Limited \\
\hline \multicolumn{5}{|l|}{ Hip } \\
\hline \multicolumn{5}{|l|}{ Sagittal Plane (flexion) } \\
\hline Jaegers et al., 1995 [9] & AMP vs INT & $5(-1$ to 11$)$ & NS & - \\
\hline Rabuffetti et al., 2005 [35] & AMP vs INT & $-14^{\dagger}$ & $\mathrm{AMP}<\mathrm{INT}$ & Limited \\
\hline \multicolumn{5}{|l|}{ Sagittal Plane (extension) } \\
\hline Jaegers et al., 1995 [9] & AMP vs INT & $7(3-11)$ & $\mathrm{AMP}>\mathrm{INT}$ & Limited \\
\hline Rabuffetti et al., 2005 [35] & AMP vs INT & $-7^{\dagger}$ & $\mathrm{AMP}<\mathrm{INT}$ & - \\
\hline \multicolumn{5}{|l|}{ Frontal Plane } \\
\hline Jaegers et al., 1995 [9] & AMP vs INT & $2(-2$ to 6$)$ & NS & Limited \\
\hline \multicolumn{5}{|c|}{$\begin{array}{l}\text { "Data extracted from graph. } \\
\text { 'Unable to calculate } 95 \% \mathrm{CI} \text { for these studies. } \\
\mathrm{AMP}=\text { amputated limb, CI = confidence interval, } \mathrm{CON}=\text { control, INT = intact limb of person with amputation, NS = not specified. }\end{array}$} \\
\hline
\end{tabular}

investigated the transverse rotation of the pelvis during walking [8,39-40]; two of those studies investigated the differences in transverse rotation between amputated and intact limb [39-40]. Both studies reported reduced pelvic transverse rotation of the amputated limb (i.e., when the amputated limb is in terminal stance) when compared with the intact limb $(p>0.05)$ [39-40]. The only study that investigated the total pelvic transverse rotation reported no difference between participants with TFA and nondisabled controls $(p>0.05)[5]$. 
Table 6.

Summary of evidence: During walking for persons with transtibial amputation.

\begin{tabular}{|c|c|c|c|c|}
\hline Study & Comparison & $\begin{array}{c}\text { Mean Difference } \\
(95 \% \text { CI })\end{array}$ & Findings & $\begin{array}{c}\text { Summary of } \\
\text { Evidence }\end{array}$ \\
\hline \multicolumn{5}{|l|}{ Pelvis } \\
\hline \multicolumn{5}{|l|}{ Frontal Plane } \\
\hline Molina Rueda et al., 2013 [47] & AMP vs CON & $-0.3(-2.4$ to 1.8$)$ & NS & Limited \\
\hline \multicolumn{5}{|l|}{ Trunk } \\
\hline \multicolumn{5}{|l|}{ Frontal Plane } \\
\hline Hendershot \& Wolf, 2014 [48] & AMP vs CON & $2(0.8-3)$ & $\mathrm{AMP}>\mathrm{CON}$ & - \\
\hline Molina Rueda et al., 2013 [47] & AMP vs CON & $2(0.2-3.6)$ & $\mathrm{AMP}>\mathrm{CON}$ & Limited \\
\hline Hendershot \& Wolf, 2014 [48] & AMP vs INT & $1.6(0.2-3)$ & $\mathrm{AMP}>\mathrm{INT}$ & - \\
\hline \multicolumn{5}{|l|}{ Hip } \\
\hline \multicolumn{5}{|l|}{ Sagittal Plane (peak flexion) } \\
\hline Kovač et al., 2010 [33] & AMP vs INT & $-1.4(-8.4$ to 5.6$)$ & NS & - \\
\hline Vanicek et al., 2009 [41] (non-fallers) & AMP vs INT & $6(-2.9$ to 14.9$)$ & NS & - \\
\hline Vanicek et al., 2009 [41] (fallers) & AMP vs INT & $0.6(-9.0$ to 10.2$)$ & NS & Limited \\
\hline Yeung et al., 2012 [45] & AMP vs INT & $-0.8(-13$ to 11.4$)$ & NS & - \\
\hline \multicolumn{5}{|l|}{ Sagittal Plane (peak extension) } \\
\hline Gates et al., 2012 [32] & AMP vs INT & $3.5(0.5-6.5)$ & $\mathrm{AMP}>\mathrm{INT}$ & Limited \\
\hline Kovač et al., 2010 [33] & AMP vs INT & $-1.2(-9.2$ to 6.8$)$ & NS & - \\
\hline \multicolumn{5}{|l|}{ Frontal Plane (peak abduction-swing) } \\
\hline Kovač et al., 2010 [33] & AMP vs INT & $3.70(0.4-7.0)$ & AMP $>$ INT & - \\
\hline Vanicek et al., 2009 [41] (fallers) & AMP vs INT & $1.2(-3.9$ to 6.3$)$ & NS & Limited \\
\hline Vanicek et al., 2009 [41] (non-fallers) & AMP vs INT & $3.9(-1.2$ to 9$)$ & NS & - \\
\hline \multicolumn{5}{|l|}{ Frontal Plane (peak adduction-stance) } \\
\hline Kovač et al., 2010 [33] & AMP vs INT & $2.6(-0.5$ to 5.7$)$ & AMP $<$ INT & - \\
\hline Vanicek et al., 2009 [41] (fallers) & AMP vs INT & $2.6(-2.7$ to 7.9$)$ & NS & Limited \\
\hline Vanicek et al., 2009 [41] (non-fallers) & AMP vs INT & $5.4(0.2-10.6)$ & $\mathrm{AMP}<\mathrm{INT}$ & - \\
\hline
\end{tabular}

Lumbar spine kinematics. Only one study investigated the lumbar spine motion between persons with TFA and nondisabled controls [18]. This study reported increased lumbar extension $\left(10^{\circ}\right)$ in participants with TFA $(n=17)$ as compared with nondisabled controls $(n=$ 6) $(p<0.05)$.

\section{Hip Kinematics During Walking}

Sagittal plane. During initial contact phase, two studies reported limited hip flexion of the amputated limb compared with the intact limb $(p>0.05)$ (Table 6) $[9,38]$. During the terminal stance phase, increased hip extension at the amputated limb $(p>0.05)$ was observed as compared with the intact limb [9]. In contrast, Rabuffetti et al. reported limited hip extension at the amputated limb compared with the intact limb $(p<0.05)$ in the terminal stance phase [35].

Frontal plane. Two studies reported hip frontal motion during walking [9,39]; only one of the studies investigated the interlimb movement differences and concluded no significant difference between the amputated and intact legs [9]. The other study graphically presented the frontal hip motion and reported that the amputated hip was held in an abducted position as compared with the intact leg throughout the stance phase of walking [39].

\section{Hip Kinematics During Other Functional Tasks}

Running. A case series of four participants investigated the differences in kinematic variables such as total hip 
sagittal motion and pelvic transverse rotation during running between amputated and intact legs [30]. Asymmetry indices for pelvis and hip joints between amputated and intact legs were reported for individual participants. Decreased pelvic transverse rotation at the amputated limb was reported as compared with the intact limb $(p<0.05)$. Decreased hip flexion at the amputated limb was reported as compared with the intact limb $(p<0.05)$, which resulted in a shorter step length during running for all four participants.

Ramp walking. On uphill walking, decreased hip flexion at the amputated limb compared with the intact limb was reported $(p<0.05)$ [44]. On downhill walking, persons with TFA compensate by increased hip extension on the amputated side compared with the intact leg $(p>$ $0.05)$.

Stair climbing. During stair descent, limited hip flexion of the prosthetic limb compared with the intact limb was reported $(p>0.05)$ [37].

Obstacle crossing. In persons with TFA, reduced hip flexion at the leading prosthetic limb was reported as compared with the leading prosthetic limb of persons with TTA $(p<0.05)$ [43].

\section{Results of Studies in Persons with Transtibial Amputation}

\section{Trunk and Lumbopelvic Kinematics During Walking}

Table 6 presents the results of individual studies and a summary of evidence.

Trunk kinematics. Frontal plane-Two studies investigated frontal trunk motion [47-48]. Both showed increased trunk lateral flexion toward the amputated side during the stance phase of the amputated limbs $(p<0.05)$ [47-48].

Pelvic kinematics. Frontal plane-Two studies investigated the side-to-side differences in pelvic obliquity $[7,47]$; there was no difference in the pelvic obliquity between the intact and amputated limb $(p>0.05)[7,47]$.

\section{Hip Kinematics During Walking}

Sagittal plane. At the hip joint, four studies investigated the sagittal hip motion during walking (Table 6) $[32-33,41,45]$. The results suggest a slight increase in peak hip extension during the stance phase as compared with the intact leg $(p>0.05)$. In terms of hip flexion, there was no difference in peak hip flexion between the amputated and intact legs.
Frontal plane. Three studies investigated hip frontal plane motion $[33,41,47]$. Studies comparing the amputated and intact legs reported that the amputated leg was held in abduction throughout the gait cycle as compared with the intact leg $(p<0.05)[33,47]$. Only one study investigated the total hip frontal motion and reported no difference between participants with TTA and the referent leg of nondisabled controls [41].

\section{Hip Kinematics During Other Functional Tasks}

Running. The only study that investigated the sagittal hip motion during running reported decreased peak hip extension during stance phase of the amputated limb $(p<0.05)$ as compared with the intact limb [36].

Ramp walking. Two studies investigated the interlimb differences in hip sagittal motion during ramp walking $[31,44]$. During ramp ascent and descent, both studies report that the hip joint of the amputated limb was less extended during late stance phase $(p>0.05)[31,44]$.

Stair climbing. During stair ascent, increased peak hip flexion was reported in the stance phase of the amputated limb $(p<0.05)$ as compared with the intact limb $[28,34,37,42]$. On stair descent, increased peak hip flexion was reported as compared with the intact leg $(p<$ $0.05)$ [28,34,37].

Obstacle crossing. Two studies compared the obstacle crossing compensatory strategies in participants with TTA and reported increased trunk forward leaning during obstacle crossing $(p>0.05)[43,46]$.

\section{DISCUSSION}

\section{Summary of Evidence}

The primary purpose of this review was to investigate the asymmetrical movements of the trunk, lumbopelvic, and hip joints in people with TFA and TTA. Walking was the most common functional task investigated in both participants with TFA and TTA. In participants with TFA, during stance phase of walking, moderate evidence exists for the presence of increased trunk lateral flexion toward the amputated limb (withinsubject comparison) $(p<0.05)[8-9,40,48]$ and increased pelvic anterior tilt $(p<0.05)[8,35,38]$. In participants with TTA, limited evidence exists for spinal, pelvic, and hip asymmetries during walking. None of the studies investigated spinal kinematics in both participants with TFA and TTA during other functional tasks (i.e., running, 
ramp walking, stair climbing, and obstacle crossing). Investigating the spinal kinematics during functional tasks other than walking will help us better understand compensatory movement strategies and the potential links to LBP in this population.

In terms of methodological quality, most of the included studies presented with limitations regarding their external validity. This was primarily due to the lack of reporting of the recruitment strategies of study participants. Poor external validity scores suggest that a convenience sample of participants was recruited for the study, which may limit the generalizability of study results. The other subscore that scored poorly in most of the included studies was the selection bias (internal validity) section. This was due to poor reporting of the timeframe during which study and control group participants were recruited for the study. Moreover, none of the studies reported a power calculation and justified their sample size, which may increase the potential for a type II error. These results concur with a similar previous review that concluded low-quality scores in the external validity and selection bias subscores [3].

\section{Spinal and Pelvic Kinematics During Walking in Persons with Lower-Limb Amputation}

Lumbopelvic and trunk asymmetries are more pronounced during the stance phase in persons with TFA. Limited evidence exists to suggest decreased pelvic obliquity on the amputated side (i.e., decreased pelvic drop on the contralateral side) as compared with the intact leg [7,39-40]. Similarly, decreased pelvic obliquity $(p>0.05)$ is reported in persons with TTA [7]. Pelvic obliquity is a normal shock-absorbing mechanism during initial stance phase of walking [7]; decreased pelvic obliquity may potentially be a compensatory strategy as a result of decreased or absent knee flexion of the amputated limb in persons with TFA and TTA [7]. Further, this movement adaptation could be due to simultaneous ipsilateral trunk flexion toward the amputated limb [47] and inability to plantarflex the prosthetic ankle [6]. Stancecontrol prosthetic knee joints (e.g., microprocessorcontrolled knee) and shock-absorbing pylons reportedly influence the pelvic obliquity by shortening the leg length during initial stance phase in people with TFA and TTA and thus warrant further investigation [7].

Among persons with TFA, during single-leg support phase (including mid-stance and terminal stance) of the amputated limb, various compensations occur at the lumbopelvic and trunk segments. Moderate evidence from the included studies suggests increased anterior pelvic tilt $(p<0.05)$ in persons with TFA as compared with nondisabled controls $[8,35,38]$. This movement pattern is suggested to compensate for restricted hip motion secondary to socket-pelvis constraints [35]. Further, this compensatory strategy is proposed to enhance prosthetic knee stability during the stance phase [38]. The only high-quality study of this review reported increased lumbar spine extension $(p<0.05)$ in persons with TFA as compared with nondisabled controls during the stance phase of walking [18]; however, the study included participants with and without a history of LBP. Nevertheless, there was no reported difference in the sagittal plane motion between the groups [18]. These results suggest that participants with TFA compensate for the limited hip motion by increasing anterior pelvic tilt and lumbar extension. Increased lumbar extension has been postulated to alter the mechanical loading of facet joints, thereby contributing to degenerative changes in the lumbar spine [49]. While conflicting evidence exists for lumbar extension as a contributing factor to LBP in the general population [49], such dynamic increase in lumbar motion during walking as a possible contributing factor to LBP warrants further research [35]. Moderate evidence from the included studies also suggests increased trunk lateral flexion $(p<0.05)$ toward the amputated limb as compared with the intact limb during single-leg support phase $[8-9,40,48]$. It has been proposed that decreased strength of the hip abductor muscles in the amputated limb is the cause for this compensatory strategy [9]; nevertheless, this claim remains untested [12]. During terminal stance of the amputated limb (i.e., simultaneous initial contact of the intact limb), findings from the graphs of the included studies suggest limited pelvic transverse rotation of the amputated limb as compared with the intact limb [7,39-40]. This compensatory strategy appears to prepare the amputated limb for swing phase [39].

\section{Hip Kinematics During Walking in Persons with Lower- Limb Amputation}

Among participants with TFA, during the preswing phase of the amputated limb (i.e., double support phase), limited evidence exists for rapid transitional movement of the hip joint from extension to flexion $(p>0.05)[9,35,38]$. The intact leg, which is in stance at the same time, appears to compensate by increasing hip and knee joint flexion. This compensatory strategy might be performed to lower the center of gravity and increase the step length of the 
intact leg [9,38]. Conflicting results exist in terms of increased or decreased hip extension of the amputated limb during terminal stance phase of walking $[9,35,38]$. The conflicting results could be due to the differences in socket designs (i.e., quadrilateral socket, ischial containment socket) of the participants. A recent study investigating the hip kinematics using different socket designs showed limited hip range of motion regardless of socket design in persons with TFA during walking [50]. In addition, hip flexor tightness and hip flexion contracture have been implicated for limited hip extension in persons with TFA [14,51]. Although nonsignificant, the findings from the included studies suggest that the hip joint of the amputated limb remains abducted throughout the stance phase, possibly because of increased stride width and compensatory trunk leaning movements $[9,39]$. Further, hip abduction contracture secondary to weak hip adductor muscles in the amputated limb could also lead to increased hip abduction during walking [51].

In participants with TTA, the hip joint of the amputated limb remains abducted throughout the gait cycle similar to participants with TFA $(p<0.05)[33,41]$. This is possibly due to ipsilateral trunk lean toward the amputated limb during stance phase [47-48]. Another common movement strategy observed in this population is increased hip extension $(p>0.05)$ during stance phase of the amputated limb. This compensatory strategy is reasoned as a protective strategy to minimize the loading of the amputated limb and as a compensation of weak knee extensor muscles $[6,10,41]$. Studies have shown decreased knee muscle strength in the amputated limb as compared with the intact limb in persons with TTA [52]. Further, to compensate for the decreased loading of the amputated limb, increased hip muscle activity at the amputated limb has also been reported [11].

\section{Hip Kinematics During Other Functional Tasks in Persons with Lower-Limb Amputation}

Overall, the compensatory strategies during various functional tasks in participants with TFA have been primarily through the hip joint of the amputated limb. Limited hip flexion of the amputated limb $(p<0.05)$ during running and uphill walking appears to result in a shorter step length [30]. Moreover, increased hip extension of the amputated limb $(p<0.05)$ during stair descending and downhill walking implies the amputated limb assumed an extended posture [44]. Whether such hip joint compensatory movements together with lumbopelvic compensatory movements (i.e., increased anterior pelvic tilt) putatively contribute to LBP warrants further investigation [12].

In participants with TTA, increased hip flexion $(p<$ $0.05)$ and limited hip extension $(p>0.05)$ of the amputated limb was reported during early and late stance phase of stair climbing and ramp walking. These results suggest that the amputated limb adopted an extended posture. Further, persons with TTA were found to compensate with anterior trunk lean $(p>0.05)$ to facilitate anterior progression during these tasks $[34,42]$. Owing to the limited prosthetic ankle range of motion in persons with TTA, the movement asymmetries observed at the amputated limb are possibly to adjust the center of mass during stair climbing and ramp walking [28]. Recent studies investigating the effects of the modified prosthetic ankle (microprocessorcontrolled ankle) during ramp walking and stair climbing appears to enhance patient safety and improve lower-limb kinematics with the modified prosthetic ankle [28,31]. Whether such improvements in lower-limb kinematics during these tasks may have an effect on lumbopelvic kinematics warrants further research.

\section{Within- and Between-Subject Comparisons}

Nine studies investigated trunk and pelvic motion [79,18,35,39-40,47-48]. Five studies investigated withinsubject differences $[7,9,35,39-40]$ and two studies utilized a nondisabled control group $[8,18]$, with two studies reporting both [47-48]. Given the complexity in measuring three-dimensional spinal motion, it is not always feasible to compare the intact and amputated sides. An alternate for that is to compare persons with amputation and nondisabled controls to provide a better understanding of normative movement patterns.

\section{Methodological Considerations}

Although most of the included studies $(n=22)$ utilized optoelectronic motion capture systems, only 13 studies employed a 6 degrees of freedom modeling strategy to calculate joint angles $[8,18,28,31-34,40-42,45,47-48]$. The other studies utilized a 3 degrees of freedom strategy $(n=$ $10)$ and 1 degree of freedom strategy $(n=2)$. The advantages of investigating joint motion via 6 degrees of freedom include lesser chances of introducing error in the data and independent tracking of joint segments [53]. Further, the included studies employed different marker sets and different modeling strategies, resulting in considerable variation in the study results (i.e., 3 vs 6 degrees of freedom). A recent study conducted in the general population 
comparing the kinematic data from different marker sets reported significant differences in the results for pelvic range of motion [53]. The results highlight the need for interpreting caution when comparing the results of different marker sets from the studies of our review [54]. In addition to different marker sets, inaccurate marker placement accounts for a major source of measurement error in computing joint kinematics [26]. Apart from one study [55], none of the included studies reported the examiner who placed the markers on the study participant, which further limits the validity and comparability of results. While most of the included studies reported placing markers in the prosthesis corresponding with the landmarks of the intact leg [8,28-31,34-39,47], this method of marker placement may not be accurate given the differences in functional mechanisms of prosthetic components [56].

Nine studies reported verification of prosthetic alignment by a certified prosthetist [8,28,31-34,40,46-47]. Optimal prosthetic alignment is important for maintaining adequate socket comfort and functioning with a prosthesis [14]. Improper prosthetic alignment has been shown to significantly increase the hip muscle activity during walking in persons with TFA [57]. While relative movements between the residual limb and socket are inevitable, no study reported whether participants report socket comfort or not during the time of testing. Uncomfortable socket fit may result in functional limb length discrepancy, potentially influencing spinal kinematics in persons with lowerlimb amputation [56]. In addition to socket fit, eight studies presented the data of residual-limb length of participants [6-9,30,32,36,46]; however, the effect of short versus long residual-limb length influencing spinal kinematics warrants further research. Comprehensive kinematic models incorporating the socket-pelvis movements during analysis could improve the validity of kinematic data [56]. In addition, considerable variation existed in the reported kinematic data in the included studies, which limited the comparability of study results. These variations included lack of description of kinematic data [38-39] and inadequate presentation of results such as presenting only graphs without actual joint data [37]. Because both kinematic and kinetic data are crucial to the understanding of movement adaptation strategies, explicit reporting and discussion of both kinetic and kinematic results are necessary to enable comparison of study results.

\section{Clinical and Research Implications}

While cross-sectional studies provide necessary information to understand the movement asymmetries [21], longitudinal studies specifically investigating the trunk and lumbopelvic movement asymmetries, such as increased trunk lateral flexion and anterior pelvic tilt during walking and other tasks (i.e., stair climbing, ramp walking, and running) over a period of time, are warranted to identify the potential causal relationship with LBP [12]. In addition, studies investigating the effects of various socket design and different types of prosthetic knee and ankle components on spinal and pelvic kinematics may have potential implications for improved prosthesis design and functional performance. In terms of methodological considerations, a need exists for developing a standardized marker placement and modeling strategy in the amputation research to enhance the validity and comparability of kinematic studies [56]. Although challenges in recruiting participants in the amputation research are evident [58], future studies should consider explicit reporting of the recruitment strategies of study participants to enhance the validity of results.

\section{Strengths and Limitations}

To our knowledge, this is the first comprehensive review investigating spinal movement asymmetries during functional tasks in persons with TFA and TTA. Nevertheless, the results of our review must be interpreted with caution. First, several definitions for the term asymmetry exist in the literature [59-60]. We opted to define asymmetry as a statistically significant difference between the amputated and intact legs or comparing the amputated and referent joint segments of nondisabled controls based on similar previous systematic reviews [3-4]. For trunk and lumbar spine segments, it may not be always feasible to compare the intact and amputated sides given the complexity of measuring spinal motion, so we deemed it appropriate to compare persons with amputation and nondisabled controls to provide a better understanding of normative movement patterns. Queries related to clinically significant difference between the joint segments of amputated and intact legs justifies further research using longitudinal study designs. Second, although two reviewers independently searched the electronic databases, we included only articles published in English owing to a limited availability of translation services, which may have introduced publication bias. Further, during title screening of articles, we excluded articles that primarily investigated kinetics during a functional task; however, kinematic data were also presented in a few of those articles [61-62], which may have limited the number of final articles for our review. A secondary search through references of the included articles and previously published reviews [3-4] strengthened our search strategy. The cause of 
amputation of participants in most of the included studies $(82 \%)$ was either trauma or tumors. The results may not be generalized to those with amputation following vascular causes such as peripheral vascular disease and diabetes, who may present with differing gait kinematics due to their slow walking velocity [63]. All of the included studies reported self-selected walking velocity of study participants, which ranged between 1.1 and $1.4 \mathrm{~m} / \mathrm{s}$. Due to differences in walking velocity, the results of those studies may bias the findings of our review. However, the values were within the acceptable limits [64-65], so we believe this may not affect the overall interpretation of our results. Due to considerable variations in the prosthetic components used in the included studies, the confounding effects of using different prosthetic components on kinematic variables may have limited our understanding of the data. Last, we excluded articles comparing the different types of prosthetic components, which may have altered our results, but we considered this to be beyond the scope of this review.

\section{CONCLUSIONS}

This review identified limited studies specifically investigating spinal and pelvic asymmetries during walking and other functional tasks in persons with TFA and TTA. Moderate evidence exists for the presence of increased trunk lateral flexion toward the amputated limb in participants with TFA together with increased anterior pelvic tilt during stance phase of walking. Limited evidence exists for the presence of spinal and pelvic asymmetries in people with TTA. In terms of other functional tasks such as running, ramp walking, stair climbing, and obstacle crossing, no study investigated spinal kinematics. While aiming to minimize movement asymmetries is never possible in this population, further cross-sectional and longitudinal studies are warranted to investigate the spinal and pelvic compensatory strategies during walking and other functional tasks and explore its potential links to LBP. Explicit reporting of participant recruitment strategy and gait data in future studies will enhance the comparability of results.

\section{ACKNOWLEDGMENTS}

\section{Author Contributions:}

Study concept and design: H. Devan, A. Carman, P. Hendrick, L. Hale, D. C. Ribeiro.
Drafting and critical revision of manuscript: H. Devan, P. Hendrick, L. Hale, D. C. Ribeiro.

Study supervision: L. Hale, D. C. Ribeiro, A. Carman.

Financial Disclosures: The authors have declared that no competing interests exist.

Funding/Support: This material was based on work supported by funding from the University of Otago Doctoral Scholarship (H. Devan). Additional Contributions: We thank Trish Leishman (librarian) for helping us in planning the search strategy. We would like to thank Parimala Kanagasabai (PhD student, University of Otago) for her assistance as a second reviewer. We would also to like to thank Professor Louise Ada for her constructive feedback on the manuscript.

\section{REFERENCES}

1. Global Lower Extremity Amputation Study Group. Epidemiology of lower extremity amputation in centres in Europe, North America and East Asia. Br J Surg. 2000; 87(3):328-37. [PMID:10718803] http://dx.doi.org/10.1046/j.1365-2168.2000.01344.x

2. Staats TB. The rehabilitation of the amputee in the developing world: A review of the literature. Prosthet Orthot Int. 1996;20(1):45-50. [PMID:8740077]

3. Prinsen EC, Nederhand MJ, Rietman JS. Adaptation strategies of the lower extremities of patients with a transtibial or transfemoral amputation during level walking: A systematic review. Arch Phys Med Rehabil. 2011;92(8):1311-25.

[PMID:21714957]

http://dx.doi.org/10.1016/j.apmr.2011.01.017

4. Sagawa Y Jr, Turcot K, Armand S, Thevenon A, Vuillerme $\mathrm{N}$, Watelain E. Biomechanics and physiological parameters during gait in lower-limb amputees: A systematic review. Gait Posture. 2011;33(4):511-26. [PMID:21392998] http://dx.doi.org/10.1016/j.gaitpost.2011.02.003

5. Nolan L, Wit A, Dudziñski K, Lees A, Lake M, Wychowañski $M$. Adjustments in gait symmetry with walking speed in transfemoral and trans-tibial amputees. Gait Posture. 2003;17(2): 142-51. [PMID:12633775] http://dx.doi.org/10.1016/S0966-6362(02)00066-8

6. Sanderson DJ, Martin PE. Lower extremity kinematic and kinetic adaptations in unilateral below-knee amputees during walking. Gait Posture. 1997;6(2):126-36. http://dx.doi.org/10.1016/S0966-6362(97)01112-0

7. Michaud SB, Gard SA, Childress DS. A preliminary investigation of pelvic obliquity patterns during gait in persons with transtibial and transfemoral amputation. J Rehabil Res Dev. 2000;37(1):1-10. [PMID:10847567]

8. Goujon-Pillet H, Sapin E, Fodé P, Lavaste F. Threedimensional motions of trunk and pelvis during transfemoral amputee gait. Arch Phys Med Rehabil. 2008;89(1): 87-94. [PMID:18164336] http://dx.doi.org/10.1016/j.apmr.2007.08.136 
9. Jaegers SM, Arendzen JH, de Jongh HJ. Prosthetic gait of unilateral transfemoral amputees: A kinematic study. Arch Phys Med Rehabil. 1995;76(8):736-43. [PMID:7632129] http://dx.doi.org/10.1016/S0003-9993(95)80528-1

10. Beyaert C, Grumillier C, Martinet N, Paysant J, André JM. Compensatory mechanism involving the knee joint of the intact limb during gait in unilateral below-knee amputees. Gait Posture. 2008;28(2):278-84. [PMID:18295487] http://dx.doi.org/10.1016/j.gaitpost.2007.12.073

11. Silverman AK, Fey NP, Portillo A, Walden JG, Bosker G, Neptune RR. Compensatory mechanisms in below-knee amputee gait in response to increasing steady-state walking speeds. Gait Posture. 2008;28(4):602-9. [PMID:18514526] http://dx.doi.org/10.1016/j.gaitpost.2008.04.005

12. Devan H, Hendrick P, Ribeiro DC, Hale LA, Carman A. Asymmetrical movements of the lumbopelvic region: Is this a potential mechanism for low back pain in people with lower limb amputation? Med Hypotheses. 2014;82(1):77-85.

[PMID:24296234] http://dx.doi.org/10.1016/j.mehy.2013.11.012

13. Morgenroth DC, Gellhorn AC, Suri P. Osteoarthritis in the disabled population: A mechanical perspective. PM R. 2012;4(5 Suppl):S20-27. [PMID:22632698] http://dx.doi.org/10.1016/j.pmrj.2012.01.003

14. Gailey R, Allen K, Castles J, Kucharik J, Roeder M. Review of secondary physical conditions associated with lower-limb amputation and long-term prosthesis use. J Rehabil Res Dev. 2008;45(1):15-29. [PMID:18566923] http://dx.doi.org/10.1682/JRRD.2006.11.0147

15. Devan H, Tumilty S, Smith C. Physical activity and lowerback pain in persons with traumatic transfemoral amputation: A national cross-sectional survey. J Rehabil Res Dev. 2012;49(10):1457-66. [PMID:23516050] http://dx.doi.org/10.1682/JRRD.2011.09.0155

16. Ehde DM, Smith DG, Czerniecki JM, Campbell KM, Malchow DM, Robinson LR. Back pain as a secondary disability in persons with lower limb amputations. Arch Phys Med Rehabil. 2001;82(6):731-34. [PMID:11387575] http://dx.doi.org/10.1053/apmr.2001.21962

17. Smith DG, Ehde DM, Legro MW, Reiber GE, del Aguila M, Boone DA. Phantom limb, residual limb, and back pain after lower extremity amputations. Clin Orthop Relat Res. 1999;(361):29-38. [PMID:10212593]

18. Morgenroth DC, Orendurff MS, Shakir A, Segal A, Shofer J, Czerniecki JM. The relationship between lumbar spine kinematics during gait and low-back pain in transfemoral amputees. Am J Phys Med Rehabil. 2010;89(8):635-43. [PMID:20647781] http://dx.doi.org/10.1097/PHM.0b013e3181e71d90

19. Friel K, Domholdt E, Smith DG. Physical and functional measures related to low back pain in individuals with lower-limb amputation: An exploratory pilot study. J Reha- bil Res Dev. 2005;42(2):155-66. [PMID:15944880]

http://dx.doi.org/10.1682/JRRD.2004.08.0090

20. Soares AS, Yamaguti EY, Mochizuki L, Amadio AC, Serrão JC. Biomechanical parameters of gait among transtibial amputees: A review. Sao Paulo Med J. 2009;127(5):302-9. [PMID:20169280]

http://dx.doi.org/10.1590/S1516-31802009000500010

21. Portney LG, Watkins MP. Foundations of clinical research: Applications to practice. 3rd ed. Upper Saddle River (NJ): Pearson/Prentice Hall; 2009. p. 277-78.

22. Downs SH, Black N. The feasibility of creating a checklist for the assessment of the methodological quality both of randomised and non-randomised studies of health care interventions. J Epidemiol Community Health. 1998;52(6): 377-84. [PMID:9764259] http://dx.doi.org/10.1136/jech.52.6.377

23. Mani R, Milosavljevic S, Sullivan SJ. The effect of occupational whole-body vibration on standing balance: A systematic review. Int J Ind Ergon. 2010;40(6):698-709. http://dx.doi.org/10.1016/j.ergon.2010.05.009

24. Higgins JP, Green S. Cochrane handbook for systematic reviews of interventions. Vol. 5. Chichester (England): Wiley-Blackwell; 2008. p. 167-80.

25. Armand S, Sangeux M, Baker R. Optimal markers' placement on the thorax for clinical gait analysis. Gait Posture. 2014;39(1):147-53. [PMID:23849985]

http://dx.doi.org/10.1016/j.gaitpost.2013.06.016

26. McGinley JL, Baker R, Wolfe R, Morris ME. The reliability of three-dimensional kinematic gait measurements: A systematic review. Gait Posture. 2009;29(3):360-69.

[PMID:19013070]

http://dx.doi.org/10.1016/j.gaitpost.2008.09.003

27. van Tulder M, Malmivaara A, Esmail R, Koes B. Exercise therapy for low back pain: A systematic review within the framework of the Cochrane collaboration back review group. Spine. 2000;25(21):2784-96. [PMID:11064524] http://dx.doi.org/10.1097/00007632-200011010-00011

28. Alimusaj M, Fradet L, Braatz F, Gerner HJ, Wolf SI. Kinematics and kinetics with an adaptive ankle foot system during stair ambulation of transtibial amputees. Gait Posture. 2009;30(3):356-63. [PMID:19616436] http://dx.doi.org/10.1016/j.gaitpost.2009.06.009

29. Bae TS, Choi K, Hong D, Mun M. Dynamic analysis of above-knee amputee gait. Clin Biomech (Bristol, Avon). 2007;22(5):557-66. [PMID:17321021]

http://dx.doi.org/10.1016/j.clinbiomech.2006.12.009

30. Burkett B, Smeathers J, Barker T. Walking and running inter-limb asymmetry for Paralympic trans-femoral amputees, a biomechanical analysis. Prosthet Orthot Int. 2003; 27(1):36-47. [PMID:12812326]

31. Fradet L, Alimusaj M, Braatz F, Wolf SI. Biomechanical analysis of ramp ambulation of transtibial amputees with 
an adaptive ankle foot system. Gait Posture. 2010;32(2): 191-98. [PMID:20457526]

http://dx.doi.org/10.1016/j.gaitpost.2010.04.011

32. Gates DH, Dingwell JB, Scott SJ, Sinitski EH, Wilken JM. Gait characteristics of individuals with transtibial amputations walking on a destabilizing rock surface. Gait Posture. 2012;36(1):33-39. [PMID:22469772]

http://dx.doi.org/10.1016/j.gaitpost.2011.12.019

33. Kovač I, Medved V, Kasović M, Heimer Ž, Lužar-Stiffler $\mathrm{V}$, Pećina M. Instrumented joint mobility analysis in traumatic transtibial amputee patients. Period Biol. 2010; 112(1):25-31.

34. Powers CM, Boyd LA, Torburn L, Perry J. Stair ambulation in persons with transtibial amputation: An analysis of the Seattle LightFoot. J Rehabil Res Dev. 1997;34(1):9-18. [PMID:9021622] http://dx.doi.org/10.1016/0966-6362(96)80615-1

35. Rabuffetti M, Recalcati M, Ferrarin M. Trans-femoral amputee gait: Socket-pelvis constraints and compensation strategies. Prosthet Orthot Int. 2005;29(2):183-92.

[PMID:16281727] http://dx.doi.org/10.1080/03093640500217182

36. Sanderson DJ, Martin PE. Joint kinetics in unilateral below-knee amputee patients during running. Arch Phys Med Rehabil. 1996;77(12):1279-85. [PMID:8976312] http://dx.doi.org/10.1016/S0003-9993(96)90193-8

37. Schmalz T, Blumentritt S, Marx B. Biomechanical analysis of stair ambulation in lower limb amputees. Gait Posture. 2007;25(2):267-78. [PMID:16725325] http://dx.doi.org/10.1016/j.gaitpost.2006.04.008

38. Sjödahl C, Jarnlo GB, Söderberg B, Persson BM. Kinematic and kinetic gait analysis in the sagittal plane of trans-femoral amputees before and after special gait re-education. Prosthet Orthot Int. 2002;26(2):101-12. [PMID:12227444] http://dx.doi.org/10.1080/03093640208726632

39. Sjödahl C, Jarnlo GB, Söderberg B, Persson BM. Pelvic motion in trans-femoral amputees in the frontal and transverse plane before and after special gait re-education. Prosthet Orthot Int. 2003;27(3):227-37. [PMID:14727704] http://dx.doi.org/10.1080/03093640308726686

40. Tazawa E. Analysis of torso movement of trans-femoral amputees during level walking. Prosthet Orthot Int. 1997; 21(2):129-40. [PMID:9285957]

41. Vanicek N, Strike S, McNaughton L, Polman R. Gait patterns in transtibial amputee fallers vs. non-fallers: Biomechanical differences during level walking. Gait Posture. 2009;29(3): 415-20. [PMID:19071021] http://dx.doi.org/10.1016/j.gaitpost.2008.10.062

42. Vanicek N, Strike SC, McNaughton L, Polman R. Lower limb kinematic and kinetic differences between transtibial amputee fallers and non-fallers. Prosthet Orthot Int. 2010;
34(4):399-410. [PMID:20450461]

http://dx.doi.org/10.3109/03093646.2010.480964

43. Vrieling AH, van Keeken HG, Schoppen T, Otten E, Halbertsma JP, Hof AL, Postema K. Obstacle crossing in lower limb amputees. Gait Posture. 2007;26(4):587-94.

[PMID:17275306]

http://dx.doi.org/10.1016/j.gaitpost.2006.12.007

44. Vrieling AH, van Keeken HG, Schoppen T, Otten E, Halbertsma JP, Hof AL, Postema K. Uphill and downhill walking in unilateral lower limb amputees. Gait Posture. 2008; 28(2):235-42. [PMID:18242995]

http://dx.doi.org/10.1016/j.gaitpost.2007.12.006

45. Yeung LF, Leung AK, Zhang M, Lee WC. Long-distance walking effects on trans-tibial amputees compensatory gait patterns and implications on prosthetic designs and training. Gait Posture. 2012;35(2):328-33. [PMID:22055554] http://dx.doi.org/10.1016/j.gaitpost.2011.10.004

46. Hill SW, Patla AE, Ishac MG, Adkin AL, Supan TJ, Barth DG. Kinematic patterns of participants with a below-knee prosthesis stepping over obstacles of various heights during locomotion. Gait Posture. 1997;6(3):186-92. http://dx.doi.org/10.1016/S0966-6362(97)01120-X

47. Molina Rueda F, Alguacil Diego IM, Molero Sánchez A, Carratalá Tejada M, Rivas Montero FM, Miangolarra Page JC. Knee and hip internal moments and upper-body kinematics in the frontal plane in unilateral transtibial amputees. Gait Posture. 2013;37(3):436-39. [PMID:23021254] http://dx.doi.org/10.1016/j.gaitpost.2012.08.019

48. Hendershot BD, Wolf EJ. Three-dimensional joint reaction forces and moments at the low back during over-ground walking in persons with unilateral lower-extremity amputation. Clin Biomech (Bristol, Avon). 2014;29(3):235-42. [PMID:24393361] http://dx.doi.org/10.1016/j.clinbiomech.2013.12.005

49. Been E, Kalichman L. Lumbar lordosis. Spine J. 2014; 14(1):87-97. [PMID:24095099] http://dx.doi.org/10.1016/j.spinee.2013.07.464

50. Klotz R, Colobert B, Botino M, Permentiers I. Influence of different types of sockets on the range of motion of the hip joint by the transfemoral amputee. Ann Phys Rehabil Med. 2011;54(7):399-410. [PMID:21996548] http://dx.doi.org/10.1016/j.rehab.2011.08.001

51. Yoo S. Complications following an amputation. Phys Med Rehabil Clin N Am. 2014;25(1):169-78. [PMID:24287246] http://dx.doi.org/10.1016/j.pmr.2013.09.003

52. Isakov $\mathrm{E}, \mathrm{Burger} \mathrm{H}$, Gregorič M, Marinček C. Isokinetic and isometric strength of the thigh muscles in below-knee amputees. Clin Biomech (Bristol, Avon). 1996;11(4):232-35. [PMID:11415626] http://dx.doi.org/10.1016/0268-0033(95)00078-X 
53. Collins TD, Ghoussayni SN, Ewins DJ, Kent JA. A six degrees-of-freedom marker set for gait analysis: Repeatability and comparison with a modified Helen Hayes set. Gait Posture. 2009;30(2):173-80. [PMID:19473844] http://dx.doi.org/10.1016/j.gaitpost.2009.04.004

54. Ferrari A, Benedetti MG, Pavan E, Frigo C, Bettinelli D, Rabuffetti M, Crenna P, Leardini A. Quantitative comparison of five current protocols in gait analysis. Gait Posture. 2008;28(2):207-16. [PMID:18206374] http://dx.doi.org/10.1016/j.gaitpost.2007.11.009

55. Morgenroth DC, Shakir A, Orendurff MS, Czerniecki JM. Low-back pain in transfemoral amputees: Is there a correlation with static or dynamic leg-length discrepancy? Am J Phys Med Rehabil. 2009;88(2):108-13. [PMID:19169176] http://dx.doi.org/10.1097/PHM.0b013e318194fbbc

56. Kent J, Franklyn-Miller A. Biomechanical models in the study of lower limb amputee kinematics: A review. Prosthet Orthot Int. 2011;35(2):124-39. [PMID:21697196] http://dx.doi.org/10.1177/0309364611407677

57. Schmalz T, Blumentritt S, Jarasch R. Energy expenditure and biomechanical characteristics of lower limb amputee gait: The influence of prosthetic alignment and different prosthetic components. Gait Posture. 2002;16(3):255-63. [PMID:12443950] http://dx.doi.org/10.1016/S0966-6362(02)00008-5

58. Hafner BJ, Sanders JE, Czerniecki J, Fergason J. Energy storage and return prostheses: Does patient perception correlate with biomechanical analysis? Clin Biomech (Bristol, Avon). 2002;17(5):325-44. [PMID:12084537] http://dx.doi.org/10.1016/S0268-0033(02)00020-7

59. Al-Eisa E, Egan D, Deluzio K, Wassersug R. Effects of pelvic asymmetry and low back pain on trunk kinematics during sitting: A comparison with standing. Spine. 2006;31(5): E135-43. [PMID:16508537] http://dx.doi.org/10.1097/01.brs.0000201325.89493.5f

60. Herzog W, Nigg BM, Read LJ, Olsson E. Asymmetries in ground reaction force patterns in normal human gait. Med Sci Sports Exerc. 1989;21(1):110-14. [PMID:2927295] http://dx.doi.org/10.1249/00005768-198902000-00020

61. Seroussi RE, Gitter A, Czerniecki JM, Weaver K. Mechanical work adaptations of above-knee amputee ambulation.
Arch Phys Med Rehabil. 1996;77(11):1209-14.

[PMID:8931539]

http://dx.doi.org/10.1016/S0003-9993(96)90151-3

62. Grumillier C, Martinet N, Paysant J, André J-M, Beyaert C. Compensatory mechanism involving the hip joint of the intact limb during gait in unilateral trans-tibial amputees. J Biomech. 2008;41(14):2926-31. [PMID:18771768] http://dx.doi.org/10.1016/j.jbiomech.2008.07.018

63. Torburn L, Powers CM, Guiterrez R, Perry J. Energy expenditure during ambulation in dysvascular and traumatic below-knee amputees: A comparison of five prosthetic feet. J Rehabil Res Dev. 1995;32(2):111-19. [PMID:7562650]

64. Cheng CK, Chen HH, Chen CS, Lee SJ. Influences of walking speed change on the lumbosacral joint force distribution. Biomed Mater Eng. 1998;8(3-4):155-65. [PMID:10065882]

65. Feipel V, De Mesmaeker T, Klein P, Rooze M. Threedimensional kinematics of the lumbar spine during treadmill walking at different speeds. Eur Spine J. 2001;10(1): 16-22. [PMID:11276830] http://dx.doi.org/10.1007/s005860000199

Submitted for publication May 27, 2014. Accepted in revised form November 10, 2014.

This article and any supplementary material should be cited as follows:

Devan H, Carman A, Hendrick P, Hale L, Ribeiro DC. Spinal, pelvic, and hip movement asymmetries in people with lower-limb amputation: Systematic review. J Rehabil Res Dev. 2015;52(1):1-20.

http://dx.doi.org/10.1682/JRRD.2014.05.0135

ResearcherID/ORCID: Hemakumar Devan, MPhty: G9585-2012; Paul Hendrick, PhD: F-7325-2012; Daniel Cury Ribeiro, PhD: G-1694-2011

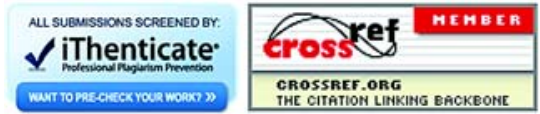


